Seguridad Operacional y Logística Aeronáutica

Segurança Operacional e Logística Aeronáutica

Operational Safety

and Aviation Logistics

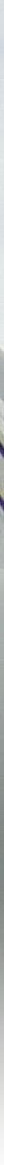

\title{
ANÁlisis de ClÚsteres aeronáuticos referentes para el desarrollo del CLÚSTER AEROESPACIAL COLOMBIANO*
}

ANÁlISE DE CLUSTERS AERONÁUTICOS REFERENTES AO DESENVOLVIMENTO DO CLUSTER AEROESPACIAL COLOMBIANO **

Analysis of aeronautical clusters referring to the deVelopment of the Colombian aerospaCe Cluster ${ }^{* * *}$

Andrés Felipe Bello Zapata

Escuela de Postgrados de la Fuerza Aérea Colombiana. Bogotá, Colombia

\section{CIENCIA Y PODER AÉREO}

ISSN 1909-7050 / E- ISSN 2389-9468 / Volumen 12/ Enero-diciembre de 2017/ Colombia/ Pp. 36-58

Recibido: 16/08/2017

Aprobado: 20/09/2017

Doi: https://doi.org/10.18667/cienciaypoderaereo.560 


\section{Para citar este artículo:}

Bello Zapata, A. F. (2017). Análisis de clústeres aeronáuticos referentes para el desarrollo del clúster aeroespacial colombiano. Ciencia y Poder Aéreo, 12, 36-58.

Doi: https://doi.org/10.18667/cienciaypoderaereo.560

* Artículo de revisión, generado como opción de grado, para obtener el título de Especialista en Logística Aeronáutica en la Escuela de Postgrados de la Fuerza Aérea Colombiana (EPFAC).

** Artigo de revisão, gerado como uma opção de graduação, para obter o título de Especialista em Logística Aeronáutica na Escola de Pós-Graduação da Força Aérea Colombiana (Escuela de Postgrados de la Fuerza Aérea Colombiana-EPFAC).

*** Review article, generated as a degree option, to obtain the title of Specialist in Aeronautical Logistics at the Postgraduate School of the Colombian Air Force (Escuela de Postgrados de la Fuerza Aérea Colombiana - EPFAC).

a Ingeniero Mecánico. Fuerza Aérea Colombiana, Escuela de Postgrados de la Fuerza Aérea Colombiana. Bogotá, Colombia. Correo electrónico: andresfb88@gmail.com
Resumen: el presente artículo de revisión, a través de una metodología analítica, realiza un análisis de siete clústeres aeronáuticos internacionales con el fin de evidenciar qué fortalezas y mejores prácticas se llevan a cabo y han sido fundamentales para lograr posicionar estos grupos empresariales entre los mejores a nivel mundial. A partir de dicho estudio, se determina cuáles de esas mejores prácticas podrían llegar a ser empleadas por parte del Clúster Aeroespacial Colombiano, considerando las capacidades adquiridas con las que cuenta a primer semestre del 2016. De igual forma, se realiza una descripción de la condición actual del Clúster Aeroespacial Colombiano, el cual es una de las instituciones líderes a nivel nacional por los logros alcanzados en materia de crecimiento y desarrollo. Finalmente, se realiza un análisis del impacto que tiene el ámbito aeronáutico en la Nación y qué ventajas podríamos Ilegar a tener si se fortaleciera mencionado sector.

Palabras clave: Clúster Aeroespacial Colombiano, clúster aeronáutico, desarrollo de clústeres.

Resumo: $O$ presente artigo de revisão, através de uma metodologia analítica, realiza uma análise de sete clusters aeronáuticos internacionais para demonstrar quais os pontos fortes e melhores práticas realizados e tem sido fundamental para conseguir posicionar esses grupos empresariais entre os melhores a nível internacional. mundo Com base neste estudo, é determinado qual dessas melhores práticas poderia ser usado pelo cluster aeroespacial colombiano, considerando as capacidades adquiridas no primeiro semestre de 2016. Da mesma forma, é feita uma descrição da condição atual cluster aeroespacial colombiano, que é uma das principais instituições em todo o país para as conquistas em termos de crescimento e desenvolvimento. Finalmente, uma análise é feita do impacto que o campo aeronáutico tem na Nação e quais as vantagens que poderiamos ter se este setor fosse fortalecido.

Palavras-chave: Cluster eroespacial Colombiano, Cluster aeronáutico, Desenvolvimento de cluster

Abstract: The purpose of this work was to document the current state of the companies, academy and government entities that make up the Valle del Cauca Aerospace Cluster, through analysis and synthesis using the qualitative method through interviews with the expert actors of the Sector selected for their knowledge and experience, with the information obtained, the diagnosis and analysis of the current state in which the sector is found through the use of the DOFA matrix, allowing the identification of the competitive strategies that will serve as input Part of the research assistance of the Doctoral Thesis titled "Technology and Innovation in the Design of a Management Model for the Development of the Valle del Cauca (Col) Aerospace Cluster" to consolidate a management proposal for the development of the Aeronautical Cluster in Cauca's Valley.

Key Words: Colombian Aeronautical Cluster, Aeronautical Cluster, Cluster's Develop 


\section{Introducción}

Un artículo de revisión es considerado como un estudio pormenorizado, selectivo y crítico, que integra la información esencial en una perspectiva unitaria y de conjunto. Es un tipo de artículo científico que, sin ser original, recopila la información más relevante de un tema específico (Carrasco, 2009). El propósito de un artículo de revisión es emplear publicaciones (artículos científicos resultado de investigación, reflexión, revisión, reportes de caso, entre otros), con el fin de establecer un punto de vista o posición frente a un tema en particular (Mora, 2013).

El presente artículo de revisión se encuentra enfocado en el sector del clúster aeronáutico en Colombia, por lo que, antes de comenzar a ahondar en el tema, es necesario definir el concepto de clúster como «[u]n sistema al que pertenecen empresas y ramas industriales que establecen vínculos de interdependencia funcional para el desarrollo de sus procesos productivos y para la obtención de determinados productos» (García, 2005).

Actualmente, la globalización industrial ha demandado la generación de estrategias empresariales con el fin de generar alianzas y acuerdos de servicio que permitan generar innovación, aplicación de nuevas tecnologías e implementación de mejores prácticas que aumenten la competitividad en los mercados internacionales. El sector aeroespacial colombiano no puede ser ajeno a esta nueva era industrial, por lo tanto, es necesario fortalecer el trabajo mancomunado a través de la generación de clústeres que integren las capacidades de compañías individuales, para generar fuertes complejos con capacidad de competir a nivel global.

La efectividad del sector aeronáutico ha sido un referente de progreso y desarrollo, al punto que diferentes países alrededor del mundo están realizando investigaciones y análisis de viabilidad de implementación de mencionado sistema, dándole la misma importancia que a áreas como la de hidrocarburos -que históricamente ha dado grandes aportes de desarrollo a las diferentes naciones-. Ejemplo de ello es el clúster industrial de COEGA, ubicado en Sur África, el cual se ha especializado en el sector de refinería de petróleo, estaciones de poder de turbinas eléctricas, procesos de outsourcing, plantas de automatización, entre otros; y actualmente se encuentra direccionando sus esfuerzos en el desarrollo aeroespacial (Albawaba, 2015).

Ahora bien, para alcanzar esa integración, el presente documento ilustra casos internacionales de desarrollo de clústeres líderes en la industria, con el fin de generar una senda que guíe el proceso que se debe llevar a cabo a ni- vel nacional para alcanzar altos estándares de calidad. De los clústeres aeronáuticos internacionales analizados en el presente artículo se describen las principales iniciativas que fueron fundamentales para el crecimiento y que pueden ser aplicables localmente.

Como se logró evidenciar en el título del presente artículo, este tiene el propósito de generar una serie de mejores prácticas que puedan llegar a ser aplicadas en la agrupación industrial, de tal manera que se fomente un crecimiento continuo a través de estrategias de benchmarking, cuyo principio radica en realizar una búsqueda de las mejores prácticas empresariales, las cuales sirven de guía para alcanzar altos estándares de calidad (Kodali, 2008). Es importante aclarar que no todas las mejores prácticas destacadas en el presente artículo están direccionadas al sector industrial, ya que es necesario que el Gobierno y la academia implementen de igual forma estrategias internacionales, teniendo en cuenta que el sector aeroespacial es como un reloj en el que se requiere que los tres engranajes mencionados trabajen de manera armónica y coordinada para su óptimo funcionamiento.

Respecto al eje central del documento, el Clúster Aeroespacial Colombiano, el presente documento realiza una descripción de la condición actual del grupo empresarial, enfatizando en las fortalezas y en los proyectos que tiene la entidad a futuro. Así mismo, se realiza una medición de la influencia que tiene el sector aeronáutico en la Nación, con el fin de resaltar y argumentar el por qué es tan indispensable que los tres integrantes de la triple alianza (Gobierno-academia-industria) ahonden en esfuerzos para hacer crecer este sector y para promover el desarrollo y el crecimiento del país.

\section{Objetivos}

El objetivo general es contrastar los clústeres aeronáuticos, referentes a nivel mundial, para definir las mejores prácticas que puedan ser aplicadas en el Clúster Aeroespacial Colombiano.

En cuanto a los objetivos específicos, lo que se propone este artículo es identificar la estructura, organización y funcionamiento de los clústeres aeronáuticos, referentes a nivel internacional. Asimismo, se pretende identificar cuál es el rol de la industria, la academia y el Gobierno en el progreso de los clústeres aeronáuticos analizados. También, realizar un diagnóstico del estado del Clúster Aeroespacial Colombiano en el 2016, y realizar la caracterización de las mejores prácticas, como herramientas para el desarrollo y fortalecimiento del Clúster Aeroespacial Colombiano. 


\section{Metodología}

\section{Marco teórico}

Considerando que el eje central del presente proyecto es el Clúster Aeroespacial Colombiano, se realiza un análisis del clúster de mantenimiento en Brasil, ya que presenta una condición muy similar, por lo que sirve como referente para el progreso del clúster local (Machado, 2013). El enfoque en el mantenimiento se realiza considerando que en dicho sector es en donde se encuentra la mayor parte de la industria de aviación nacional.

Otro caso ejemplar de progreso y desarrollo, analizado en el presente artículo, hace referencia al Clúster Aeroespacial Mexicano; se realiza un análisis basado en la implementación de estrategias industriales que involucran la triple alianza (Gobierno-academia-industria), fomentando el trabajo mancomunado para el éxito del sector aeronáutico (Obregón, 2014).

El sector aeronáutico actualmente se encuentra liderado por las dos principales industrias mundiales: Boeing y Airbus. Cada una de las empresas relacionadas se sustenta con una cantidad de clústeres considerable debido a la complejidad y al arduo esfuerzo que conlleva la producción de aeronaves a gran escala (Aguilera, 2010). Es por esto que se ha reservado en el documento un espacio para analizar el desarrollo del Clúster Español, pues es muestra de una serie de iniciativas innovadoras que han hecho de la agrupación empresarial una gran industria, con capacidad para soportar los enormes retos generados por parte de las productoras de aviones de última generación a nivel mundial.

Después del análisis de las iniciativas implementadas en las diferentes áreas del mundo, se hace necesario evaluar cuál es la condición del sector aeroespacial a nivel nacional (Mesa, 2016). Es por este motivo que dentro del contenido se contempla analizar cuál es la condición del Clúster Aeroespacial Colombiano, y analizar cuál es el impacto de la industria aeronáutica en la Nación.

\section{Marco conceptual}

La tecnología y las herramientas con las que actualmente cuenta la sociedad, tales como el internet, han permitido unir al mundo tanto a nivel político, como económico, social, comercial, etc (Mona V. Makhija, 1997). La globalización ha generado diversas mutaciones en el desarrollo y crecimiento del sector industrial, al brindar nuevas expectativas a los clientes, lo cual los hace más exigentes a la hora de demandar un producto o servicio.

Con el fin de asumir y cumplir con los retos que la sociedad demanda cada día, las empresas han abundado en múltiples estrategias e iniciativas comerciales y tecnológicas que los ayuden a ser más competitivos. Durante esta carrera contra el tiempo y el dinero, muchas de las empresas se han encontrado con barreras que han impedido el crecimiento esperado en diferentes áreas, como lo han sido el factor tecnológico, el del talento humano, el económico (know-how), la materia prima, entre otros.

La industria entonces cambió el enfoque e inicio la búsqueda de nuevas alternativas para alcanzar el nivel de competitividad que los clientes y la globalización estaba demandando. Fue allí donde se iniciaron las alianzas entre empresas, inicialmente para aspectos de información (knowhow); pero, con el tiempo, a medida que se fue adquiriendo confianza, mediante acuerdos de propiedad intelectual, se fue mejorando la sinergia de las pequeñas alianzas (Liu, 2009). Así mismo, esas pequeñas alianzas se convirtieron en grandes agrupaciones industriales, tanto internas (nacionales), como externas (internacionales) y combinadas.

La generación de agrupaciones empresariales fue un fenómeno fuertemente adoptado por la industria aeronáutica, considerando que es uno de los sectores a los que más se les ha demandado crecimiento y desarrollo por el impacto que este genera en los diversos campos: turismo, sector espacial, comercial, industrial, militar, político, entre otros. Desde las grandes empresas (Boeing, Airbus), hasta los talleres aeronáuticos, iniciaron la formación de agrupaciones empresariales (clústeres aeronáuticos) tanto a nivel nacional, como a nivel internacional (Airbus, 2009). Lo anterior se refleja, por ejemplo, en la fabricación del Airbus 320, el cual tiene una producción internacional a través de la integración de diferentes empresas en cuatro países (Francia, España, Alemania y Reino Unido).

El sector aeroespacial se ha caracterizado desde sus inicios por ser un área que involucra los últimos avances tecnológicos para su óptimo funcionamiento, además de fomentar cada vez más la implementación de iniciativas innovadoras tendientes a mejorar la productividad del mundo aeronáutico (Kehayas, 2007). Para ello es necesario que se desarrolle un trabajo mancomunado, ya que el sector industrial por sí solo no se encuentra en capacidad de cumplir con las exigencias que integra el gran mundo de la aviación.

Este es el motivo por el cual el sector aeronáutico requiere que la industria se alíe con el Gobierno y la academia en lo que comúnmente se conoce como la «Triple Alianza» (Obregón, 2014). Dentro de este engranaje, el Gobierno juega un papel fundamental, ya que está en capacidad de generar políticas que favorezcan al sector, tales como tratados de libre comercio con países estratégicos, 
normatividades aduaneras favorables, acuerdos de cooperación internacional, reglamentaciones industriales, vías, aeropuertos, muelles, seguridad, ente otros.

Así mismo, la academia tiene un rol indispensable en este proceso, ya que, a través de sus centros de investigación y laboratorios, está en capacidad de brindar apoyo a las industrias para diferentes aspectos como: caracterización de materiales, innovación de materiales, métodos de producción, etc. (Mesa, 2016). Otro papel fundamental de la institución educativa es la formación del talento humano que liderará los procesos industriales, para lo cual es necesario generar programas que estén relacionados con el tema aeroespacial (Obregón, 2014).

\section{Marco legal}

El presente artículo de revisión está enmarcado dentro del ámbito aeronáutico, especialmente en lo referente a la industria que lo promueve. Para ello, existe una gran cantidad de normatividades, tanto nacionales como internacionales, que la rigen, como se relaciona a continuación.

El RAC (Reglamentos Aeronáuticos de Colombia), en su parte 21, hace referencia a la «Certificación de aeronaves y componentes de aeronaves», normatividad que debe ser ampliamente contemplada por las empresas pertenecientes a los diferentes clústeres con el fin de lograr la certificación para los productos y servicios prestados (RAC, 2015).

La FAA (Federal Aviation Administration), en la parte 21 de su reglamento, hace referencia a «Certification procedures for products and articles», es decir, a los diferentes procedimientos que se deben llevar a cabo para la certificación de componentes aeronáuticos (FAA 2. , 2009).

Asimismo, en la parte 39 menciona las «Airworthiness directives», las diferentes directivas que tiene establecida la FAA con respecto a las aeronaves, tren de potencia, motores y los diferentes accesorios (FAA 3., 2002).

La ICAO (International Civil Aviation Organization), en su anexo no. 8, «Airworthiness of aircraft», establece las normatividades para el diseño, construcción y sus debidos certificados, con el fin de garantizar seguridad en la operación (ICAO 8. , 2010).

La Aeronáutica Civil tiene contemplado un Plan de Navegación Aérea, el cual formula los lineamientos estratégicos del 2014 al 2025, para la aviación colombiana (UAEAC, 2014).

El Decreto 2937 del 2010, generado por el Ministerio de Transporte, establece que la autoridad aeronáutica en el país es la Fuerza Aérea Colombiana para las fuerzas del
Estado, además de representar el ente coordinador con la autoridad aeronáutica civil colombiana (Ministerio de Transporte, 2010).

\section{Análisis de clústeres aeronáuticos}

\section{Desarrollo de clústeres internacionales}

En la presente sección se realiza la descripción de los clústeres internacionales referentes analizados (Brasil, España, México, Reino Unido y Francia), de los cuales se extrajeron las mejores prácticas. El criterio para la escogencia de estos clústeres se basó en la aplicabilidad y en las iniciativas que cada una de las agrupaciones empresariales implementa.

\section{Desarrollo del clúster aeroespacial HELICE, España}

Un clúster generalmente parte del hecho de que una empresa empieza a presentar un progreso que demanda la interacción con otras industrias con el fin de lograr satisfacer las nuevas necesidades de los clientes (Aguilera, 2010). Lo anterior a la vez estimula el proceso de investigación y desarrollo, abriendo las puertas al ingreso de nuevos servicios y capacidades.

El presente análisis desarrolla el origen de los clústeres aeronáuticos, en relación con la capacidad industrial, para satisfacer las altas demandas de los clientes. El clúster HELICE se consideró como referente ya que representa uno de los grupos industriales más productivos en el continente europeo. La creación de la alianza se remonta a 1990, cuando un grupo de empresas en Andalucía decidieron aliarse para adquirir mayor competitividad. La asociación cumplió rápidamente su meta de llegar a ser conocida a nivel mundial, llegando a producir el $40 \%$ de los componentes de grandes industrias aeronáuticas como Boeing y Dornier (Aguilera, 2010).

Una vez realizado el lanzamiento del clúster, se dio inicio al apoyo gubernamental a través de metodologías de crecimiento del mercado nacional (PIMA, 2005). El estado de Andalucía aumentó la competitividad de la sociedad industrial a nivel europeo, además dispuso de una infraestructura localizada estratégicamente.

Otro engranaje fundamental empleado fue la academia, pilar fundamental de la denominada triple alianza Gobierno-industria-universidad (Rosenfeld, 1997). La Universidad de Sevilla impulsó la investigación, innovación y desarrollo académico del clúster mediante la creación de foros que atrajeron profesionales de múltiples disciplinas relacionadas con el sector. 
Se logró establecer un clúster aeronáutico conformado por 39 entidades, con acuerdos de servicios externos (EADS-CASA y subcontratistas), instituciones de apoyo (centros de investigación, universidades, Gobierno) y alianzas estratégicas, logrando un incremento en la calidad de los servicios (Africa Ariño, 1997), siendo indispensable el apoyo estatal.

La organización del Clúster de Andalucía está basada en «patronatos», los cuales están conformados por los promotores del sector aeroespacial: industria, academia, Gobierno, cámaras de comercio, sindicatos, entre otros; encabezados por un presidente y vicepresidente (HELICE, 2016).

A continuación, se relaciona la gestión del clúster con la nueva era económica, denominada como la era del conocimiento (Antonelli, 1999), con la finalidad de identificar el flujo de información y analizar las implicaciones que tienen los límites geográficos en la transferencia de conocimiento.

La dinámica de la información está compuesta por dos tipos de conocimiento: conocimiento tácito y conocimiento explícito (Otero, 2015). Este último puede ser codificado, por lo cual presenta gran facilidad para su transferencia, sin importar la zona geográfica; mientras que el conocimiento tácito está incorporado en la industria a través de la rutinas y habilidades de los trabajadores, haciendo difícil intercambiar la información a grandes distancias.

Existen dos medios principales para la adquisición de la información: La primera es denominada «adquisición voluntaria» y se obtiene mediante la creación de marcos de cooperación (know-how); o a través de «adquisiciones no voluntarias» generadas a raíz de las diferentes contrataciones/ despidos que involucran un flujo de personal (Ingram, 2000).

En la industria aeronáutica, la generación de clústeres se define a través de decisiones estratégicas gubernamentales, mezcladas con planes de desarrollo nacional (Otero, 2015). Otra cualidad de esta industria es su definida jerarquización (Cooke, 1998), donde la organización piramidal presenta en su más alto nivel a las CIF (Compañías Integradoras Finales), representadas por macroempresas (Boeing, Airbus,); en el segundo nivel se ubican los Tier 1, desarrolladoras componentes (propulsión, aviónica, comunicaciones); y, en último nivel, se ubican los Tier 2 y 3, los cuales se encargan de realizar trabajos más especializados (WestWorld, 2014).

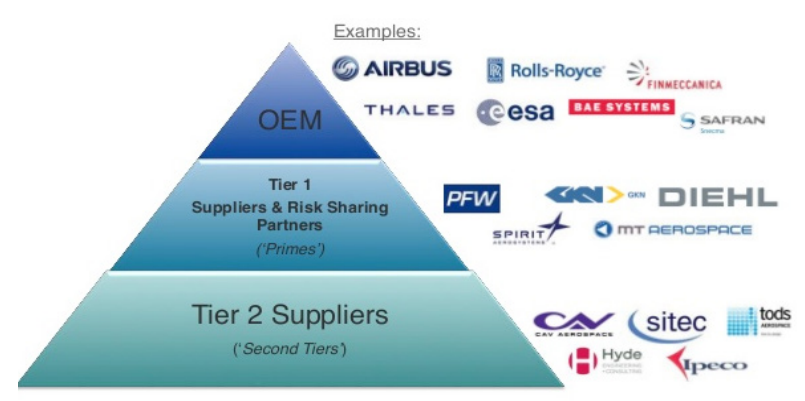

Figura 1. WestWorld (2014). Estructura Clúster Aeronáutico GeneralFuente: http://www.slideshare.net/westworldconsulting/webinar-accessing-the-european-aerospace-market-and-supply-chain

La imagen anterior ilustra que el flujo de información es direccionado de las empresas (CIF-OEM) hacia las Tier 2-3, fomentando de esta manera la cooperación tecnológica. Las empresas que generan una mayor cantidad de conocimiento, investigación y desarrollo son las ubicadas en el CIF-OEM y el TIER 1 (Otero, 2015). Dado que el sector aeroespacial tiene relación con el sector militar, se genera un alto nivel de reserva en la difusión de la información.

Otro factor influyente es el referente a las distancias geográficas existentes entre las entidades, considerando que las externalidades de conocimiento son sensibles a las distancias geográficas (Geuna, 2001). Por esta razón se hace más efectiva la transferencia de información cuando se realiza de manera nacional, ya que se ejecuta en un mismo entorno cultural.

\section{Clúster de mantenimiento aeronáutico, Brasil}

La organización de las empresas en dicha región comienza con la formación de un clúster aeronáutico gestionado por la Administración Nacional de Aviación Civil (ANAC, 2005). La ANAC cuenta con normativas claras orientadas a fortalecer la industria, dentro de las que se destacan la Regulación de Aviación Civil de Brasil RBHA 145 (Brazilian Civil Regulation, 2005) y la regulación RBHA 135 (Brazilian Civil Regulation, 2003).

Además de recibir la información, el ANAC realiza un análisis del campo de acción de cada una de las empresas certificadas (Machado, 2013). Este análisis tiene como finalidad evitar que las empresas presten servicios para los cuales no se encuentran capacitados, además de generar una base de datos que permita facilitar la comercialización.

La Tabla 1 ilustra las cinco categorías y catorce subcategorías que implementó el ANAC para clasificar a las empresas certificadas en labores de mantenimiento dentro de los diferentes clústeres aeronáuticos en la nación (Durand, 2008). 
Tabla 1.

Clasificación de certificación ANAC

\section{Pattern}

Pattern C - Maintenance, modification and cells repair

\section{Apoyos aéreos}

(C1) - Composite Structure Aircfraft, with maximum approved takeoff weight up to 5670 Kg (Aircfraft) or $2730 \mathrm{Kg}$ (Helicopters) per aircraft model.

(C2) - Metal Structure aircfraft, with maximum approved takeoff wight up to $5670 \mathrm{Kg}$ (Aircfraft) or $2730 \mathrm{Kg}$ (Helicopters) per aircraft model.

(C3) - Composite structure aircraft, with maximum approved takeoff wight over $5670 \mathrm{Kg}$ (Aircfraft) or $2730 \mathrm{Kg}$ (Helicopters) per aircraft model.

(C4) - Metal structure aircraft, with maximum approved takeoff wight over $5670 \mathrm{Kg}$ (Aircfraft) or $2730 \mathrm{Kg}$ (Helicopters) per aircraft model.

Pattern D Maintenance, modification and aircfraft engines repair

(D1) - Conventional egines with up to 400 H.P per model.

(D2) - Conventional egines with over 400 H.P per model.

(D3) - Turbine engines, per model
Pattern E Maintenance, modification and aircfraft propellers and rotors repair.

Pattern F - Maintenance, modification and aircfraft propellers and rotors repair.

Pattern $\mathrm{H}$ - Specialized service

(E1) - Wood propellers, metal or composite, fixed pitch, per model.

(E2) - All other propellers, per model.

(E3) - Helicopters rotor, per model.

(F1) - Communications and navigation aircraft equipment, per model.

(F2) - Aircraft instruments, per instrument type.

(F3) - Mechanical accessories, aircraft electrical and electronics, per accessory model.

Single Class - Specific activities for the maintenance implementation that aeronautical authority upheld, per type service (e.g; nondestructive testing, floats, emergency equipment, rotor shovels, screen coating).

Fuente: Cardoso Machado. Clustering The Technical Capability in The Brazilian Aeronautical Maintenance Industry, 2013.

La Tabla 2 ilustra dos matrices de certificación elaboradas por el ANAC para definir el número de empresas máximo por clasificación. Basado en dicho número máximo, se define cuántas empresas presentan certificados mixtos (más de una certificación). El modelo matemático se realiza basado en evitar la superposición (Philippe De Bruecker, 2015). En la Tabla 3 se evidencia una relación del número de capacidades en una escala de 0 a 1, en donde 1 representa el número máximo de empresas.

La estructura implementada por el presente clúster es muy similar al evidenciado en el clúster español, el cual se encuentra clasificado según la capacidad de las empresas, encontrándose en el sector superior de la pirámide los CIF y TIER 1, 2 y 3.

Tabla 2

Matriz de certificación combinada

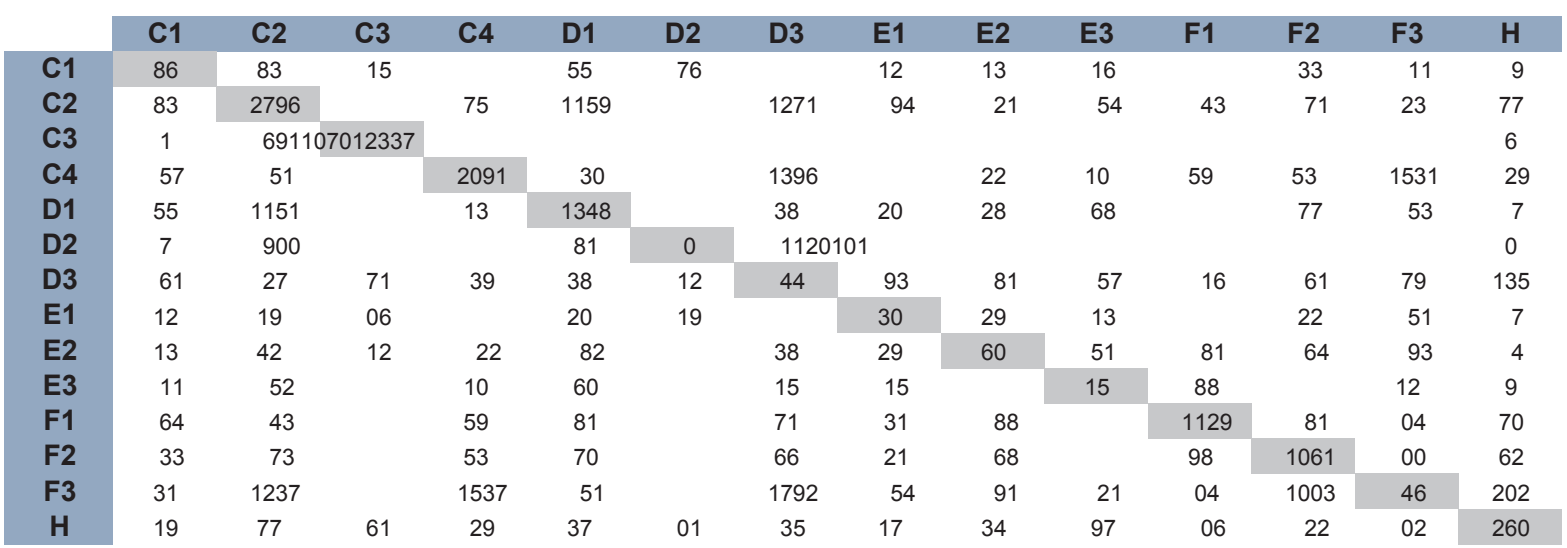

Fuente: Cardoso Machado. Clustering The Technical Capability in The Brazilian Aeronautical Maintenance Industry, 2013. 
Tabla 3.

Estandarización matriz de certificación combinada

\begin{tabular}{|c|c|c|c|c|c|c|c|c|c|c|c|c|c|c|}
\hline & C1 & C2 & C3 & C4 & D1 & D2 & D3 & E1 & E2 & E3 & F1 & F2 & F3 & $\mathrm{H}$ \\
\hline C1 & 1,000 & 0,965 & 0,0120 &, 058 & 0,640 & 0,081 & 0,0700 & 1400 & ,151 & 0,012 & 0,070 & 0,0350 & ,360 & 0,221 \\
\hline $\mathrm{C} 2$ & 0,297 & 1,000 & 0,0220 & ,269 & 0,412 & 0,032 & 0,455 & 0,0680 & ,151 & 0,054 & 0,158 & 0,1330 & ,4410 & ,276 \\
\hline C3 & 0,111 & 0,667 & 1,000 & 0,111 & 0,111 & 0,000 & 0,778 & 0,0000 & ,111 & 0,222 & 0,333 & 0,333 & 0,778 & 0,667 \\
\hline C4 & 0,024 & 0,3590 & ,005 & 1,000 & 0,062 & 0,000 & 0,6650 & ,029 & 0,105 & 0,048 & 0,282 & 0,254 & 0,7320 & ,617 \\
\hline D1 & 0,410 & 0,858 & 0,0070 & ,097 & 1,000 & 0,060 & 0,284 & 0,1490 & ,209 & 0,045 & 0,060 & 0,0520 &, 560 & 0,276 \\
\hline D2 & 0,700 & 0,900 & 0,0000 & ,000 & 0,800 & 1,000 & 0,1000 & ,100 & 0,200 & 0,000 & 0,1000 &, 000 & 0,1000 & ,000 \\
\hline D3 & 0,025 & 0,5200 &, 029 & 0,570 & 0,156 & 0,004 & 1,000 & 0,0370 & ,156 & 0,061 & 0,291 & 0,2700 &, 734 & 0,553 \\
\hline E1 & 0,400 & 0,6330 & ,000 & 0,200 & 0,667 & 0,033 & 0,300 & 1,0000 & ,967 & 0,033 & 0,1000 &, 067 & 0,833 & 0,567 \\
\hline E2 & 0,217 & 0,7000 &, 017 & 0,367 & 0,467 & 0,033 & 0,6330 &, 483 & 1,000 & 0,083 & 0,3000 &, 267 & 0,817 & 0,567 \\
\hline E3 & 0,067 & 1,000 & 0,1330 &, 667 & 0,400 & 0,000 & 1,000 & 0,0670 & ,333 & 1,000 & 0,533 & 0,533 & 0,800 & 0,600 \\
\hline F1 & 0,054 & 0,3930 &, 027 & 0,527 & 0,071 & 0,009 & 0,634 & 0,0270 & ,161 & 0,071 & 1,000 & 0,8750 & ,929 & 0,625 \\
\hline F2 & 0,028 & 0,3490 & ,028 & 0,500 & 0,066 & 0,000 & 0,6230 &, 019 & 0,151 & 0,075 & 0,925 & 1,0000 &, 943 & 0,585 \\
\hline F3 & 0,090 & 0,3550 & ,020 & 0,442 & 0,217 & 0,003 & 0,5170 & ,072 & 0,142 & 0,035 & 0,301 & 0,289 & 1,000 & 0,584 \\
\hline $\mathrm{H}$ & 0,073 & 0,2960 & ,023 & 0,496 & 0,142 & 0,000 & 0,5190 & ,065 & 0,131 & 0,035 & 0,269 & 0,238 & 0,7771 &, 000 \\
\hline
\end{tabular}

Fuente: Cardoso Machado. Clustering The Technical Capability in The Brazilian Aeronautical Maintenance Industry, 2013.

\section{Clúster aeronáutico México}

El interés en el clúster mexicano parte de que ha sido un sector potencial que ha presentado un crecimiento anual de $17,2 \%$, cuenta actualmente con un total de 287 empresas y emplea a más de 32.600 profesionales de alto nivel, lo que lo ha llevado a obtener un valor de 5.463 millones de dólares en el año 2013 (Obregón, 2014).

\section{Millones de dólares}

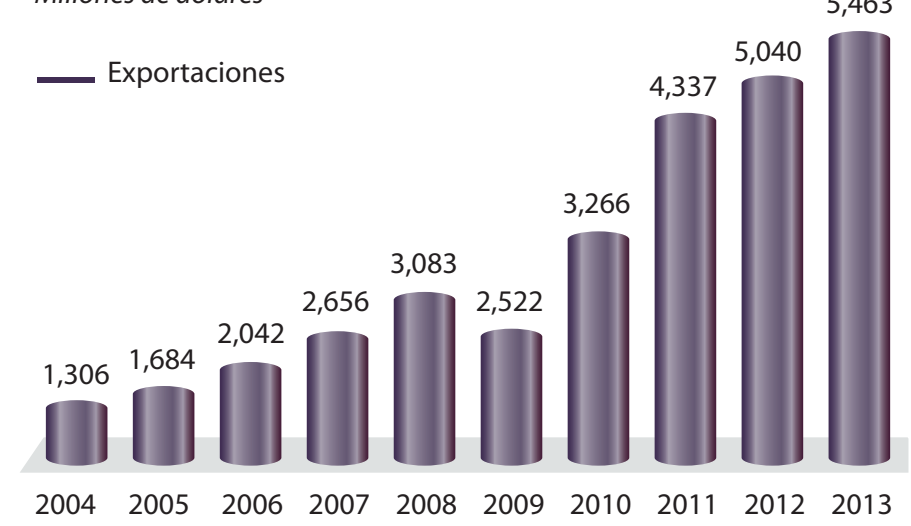

Figura 2. Exportaciones aeroespaciales mexicanas. Fuente: Obregón (2014).

La industria aeroespacial mexicana inicia en los años 50 y 90 mediante una serie de esfuerzos sin éxito, ejecutados por entidades estatales no especializadas en el sector, las cuales realizaron experimentos poco efectivos. Fue hasta el año 2010 que el sector tuvo éxito, a raíz de la creación de la Agencia Espacial Mexicana (AEM, 2010).

La nación, al forjar su vocación como centro de manufactura, tener un alto grado de tecnológico, alta calidad en la mano de obra y capacidad área de ingeniería, ha per- mitido que empresas internacionales (Bombardier, Grupo Safran, General Electric (GE), Honeywell y Eurocopter) hayan encontrado las condiciones para desarrollar centros de diseño, producción e ingeniería (Ornelas, 2007).

Los principales actores de la comunidad espacial realizan reuniones periódicas para direccionar las áreas de desarrollo y promover el crecimiento de la industria (PROMEXICO, 2007). Las estrategias e hitos que resultan de las reuniones definen el PVN (Plan de Vuelo Nacional), el cual estipula las metas estratégicas, basadas en análisis de las tendencias globales.

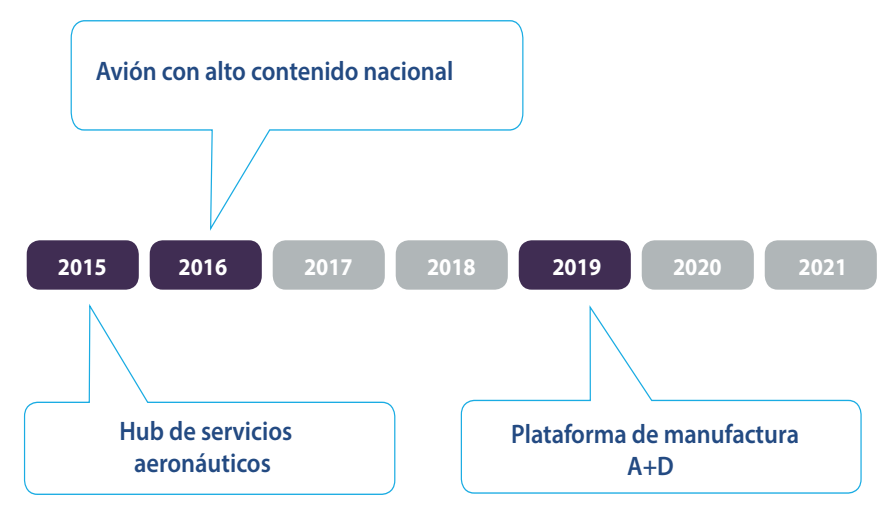

Figura 3. Hitos estratégicos

Fuente: Obregón (2014).

Para garantizar los altos estándares de calidad, el Gobierno cuenta con un instituto llamado Sistema Nacional de Calidad (CONACIT, 1970). Dicha entidad tiene la misión de administrar la certificación, acreditación e implementación de mejores prácticas, que constituyen la base para la generación de empresas de alta calidad, satisfaciendo el complejo mercado internacional. 
Otra importante gestión fue el Acuerdo Bilateral de Seguridad Aérea (BASA) (Gobierno Mexicano, 2003), ya que representa un reconocimiento de parte de la FAA al Gobierno mexicano, para la certificación de componentes, e inclusive la certificación de una aeronave completa. Actualmente, el Gobierno se encuentra tras la certificación para el desarrollo de procesos de mantenimiento y reparación (MRO), con el fin de intervenir el ciclo completo de una aeronave.

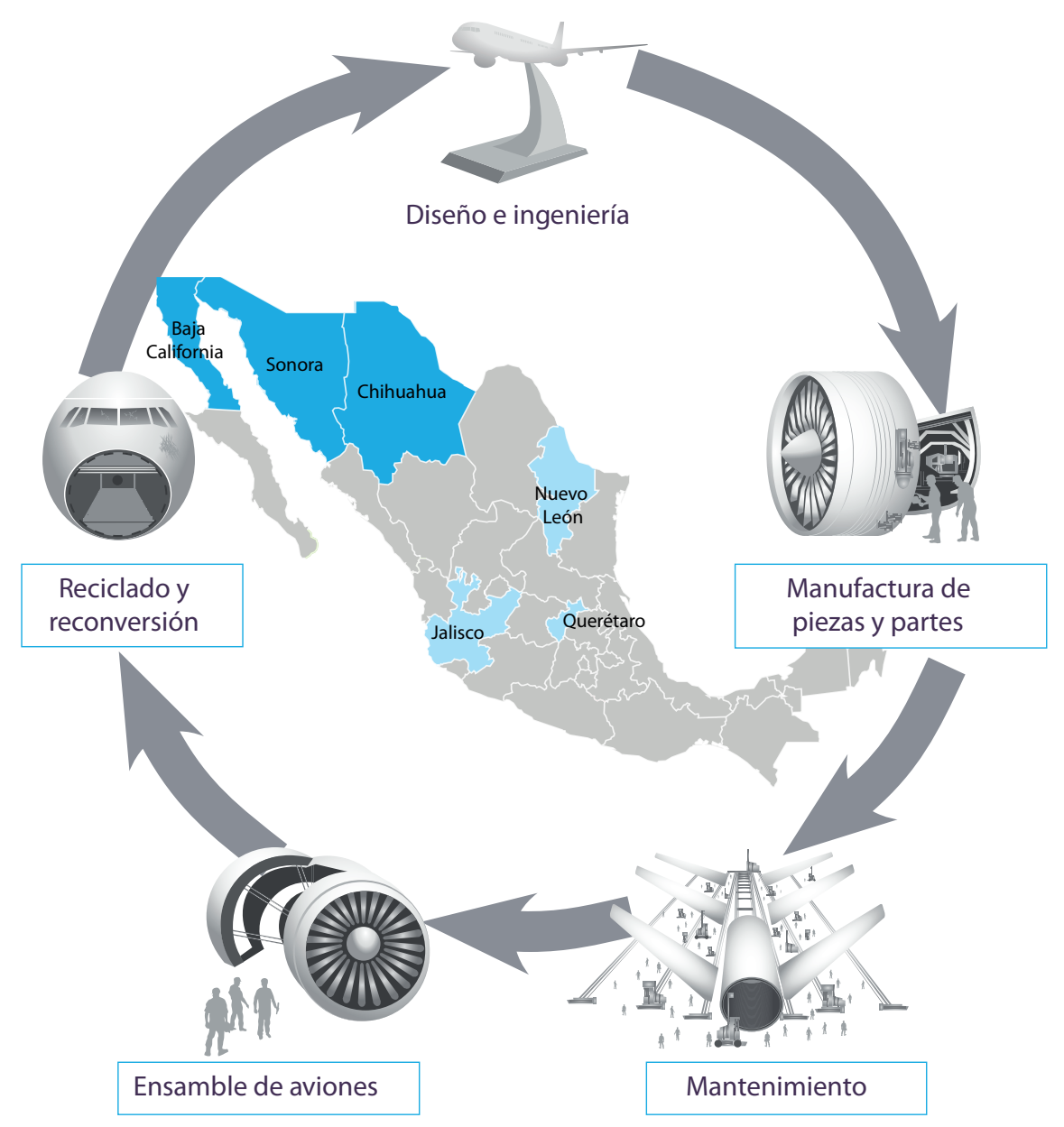

Figura 4. Ciclo de un Aeronave

Fuente: Obregón (2014).

Es de destacar la creación de un grupo intersecretarial que se encarga de identificar posibilidades de atracción de inversión y comercio internacional en los diferentes tipos de mercados, basándose en el sector de la defensa y alta tecnología (Secretaría Mexicana, 2012). Esto se logró mediante el ingreso oficial al arreglo Wassenaar (2012), el cual contribuye a la seguridad y estabilidad mediante el control de importaciones.

De igual forma, el Gobierno mexicano ha gestionado el ingreso a otros convenios como lo son: Grupo de Suministradores Nucleares (NSG, 2013), que ha permitido operar en un ambiente de mayor seguridad, acompañado de un fortalecimiento de la plataforma industrial; convenio de Grupo Australia (Group, 2013), el cual fortaleció lo referen- te al comercio de sustancias químicas, agentes biológicos, entre otros.

Otro aspecto que ha llamado la atención de los hemisferios globales es el acompañamiento de autoridades civiles aeronáuticas que impulsaron acuerdos con instituciones aeronáuticas (FAA) y los tratados de exportación cómodos y seguros (Carrillo, 2005), a través de los siguientes mecanismos de intervención:

\section{IMMEX}

Permite la importación temporal de bienes destinados a la elaboración, transformación o reparación de mercancía para su exportación sin cubrir el pago de impuestos por el ingreso al país (Secretaría de Economía, 2006). 


\section{Draw back}

Permite, según el tipo de material importado, recuperar el importe del impuesto pagado por parte de la empresa (Comercio y Aduanas, 2001).

El Gobierno creó las denominadas ZEE (Zonas Económicas Especiales), las cuales ofrecen incentivos a los inversionistas externos mediante altas expectativas económicas, exenciones fiscales, condiciones favorables de infraestructura, facilidades administrativas, mano de obra calificada, entre otros (González, 2014).

El Gobierno también implementó una simplificación arancelaria, un planteamiento de esquemas de excepción y la creación de fracciones arancelarias que facilitó la comercialización de productos y materia prima para los procesos de producción (Obregón, 2014). Otra estrategia clave fue las negociaciones Offset (Nohora Eugenia, 2002), aprovechadas para promover el desarrollo industrial y explotar la competitividad de los proyectos estratégicos.

Asimismo, se desarrollaron Centros Integrales de Servicio Aeronáutico, los cuales ofrecen un ecosistema que desarrolla la industria, estableciendo zonas comerciales preferenciales y formación en el sector aeroespacial (Obregón, 2014). Se innovó también mediante la creación de parques especializados que facilitaron las transacciones y logística de las empresas, a su vez que desarrollaron procedimientos estándar internacionales (Maldonado, 2009).

Por otro lado, se generó un progreso en la academia mediante la integración de instituciones educativas aeroespaciales (pilotos, tripulantes, técnicos, etc.) con el fin de crear un espacio de entrenamiento para el desarrollo del talento humano (Obregón, 2014). Para ello se analizaron tanto los aspectos de necesidades de la industria aeroespacial, como la oferta. A continuación, se ilustran gráficos que ilustran el comportamiento del flujo de personal.

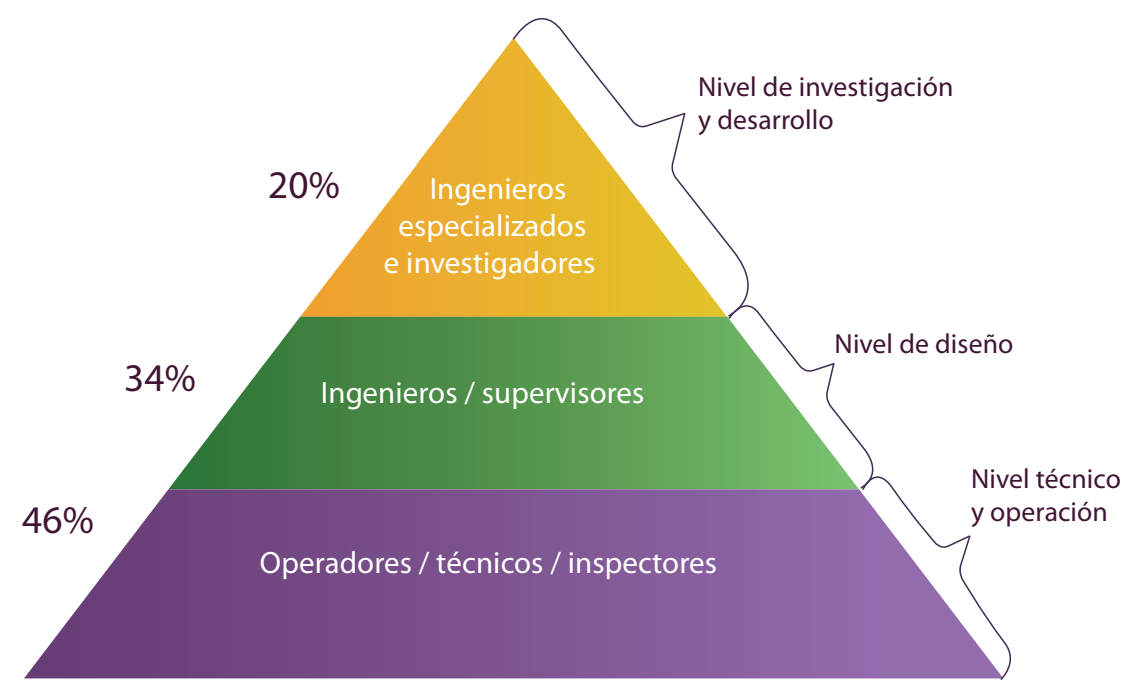

Figura 5. Pirámide de Demanda Educativa, Recuperado de Industria Aeroespacial Mexicana Mapa De Ruta 2014 Fuente: Obregon (2014). 


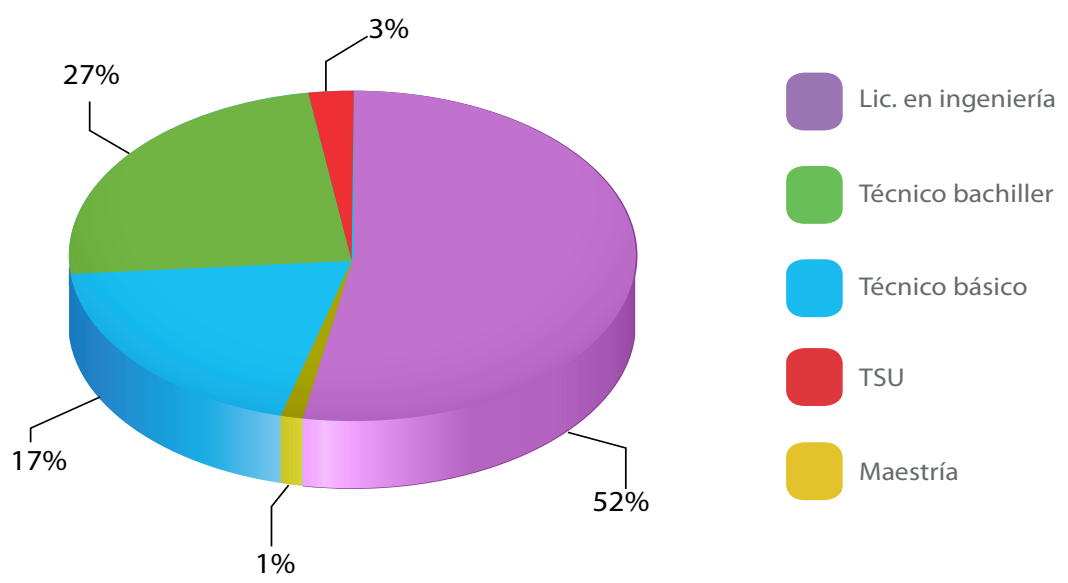

Figura 6. Oferta Educativa para la Industria Aeroespacial.

Fuente: Obregón (2014).

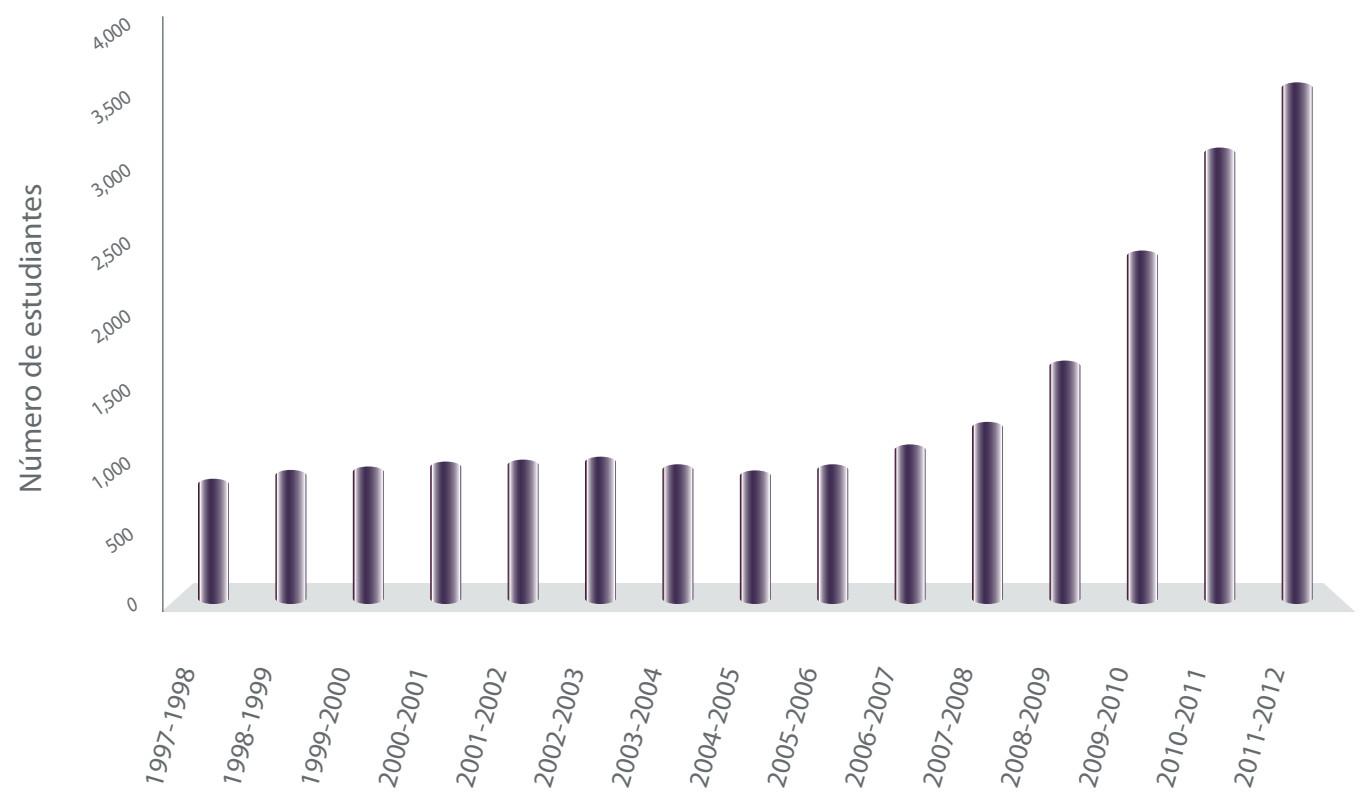

Ciclo escolar

Figura 7. Inscripción en Ingeniería Aeronáutica / Espacial

Fuente: Obregón (2014).

Actualmente se está realizando un esfuerzo en el cuidado del medio ambiente, por tanto, como estrategia de alineación con las políticas globales de reducción de la contaminación, el Gobierno se vinculó al AFRA (Asociación de Reciclaje de Flota de Aeronaves), para implementar modelos de producción que permitan reutilizar las piezas de las aeronaves (AFRA, 2005).

Otra metodología que se desarrolló fue la implementación de estrategias con el propósito de incentivar polos de competitividad e identificar industrias potenciales. Para ello, a las empresas que participan se les da acceso a bases de datos extensas de proveedores, servicios especializados, fuentes de talento humano, conocimiento, entre otros (Obregón, 2014).

Ya habiéndose consolidado como una industria de apoyo considerable para empresas aeroespaciales, actualmente esta industria tiene el reto de producir un prototipo generado en un $100 \%$ a partir de las capacidades adquiridas por cada uno de los clústeres (Obregón, 2014); dicho hito (Figura 8.), marcará un gran salto para la carrera aeroespacial. 
Baja California es el pricipal hub de exportación de servicios KPO para la industria de A+D en el país.

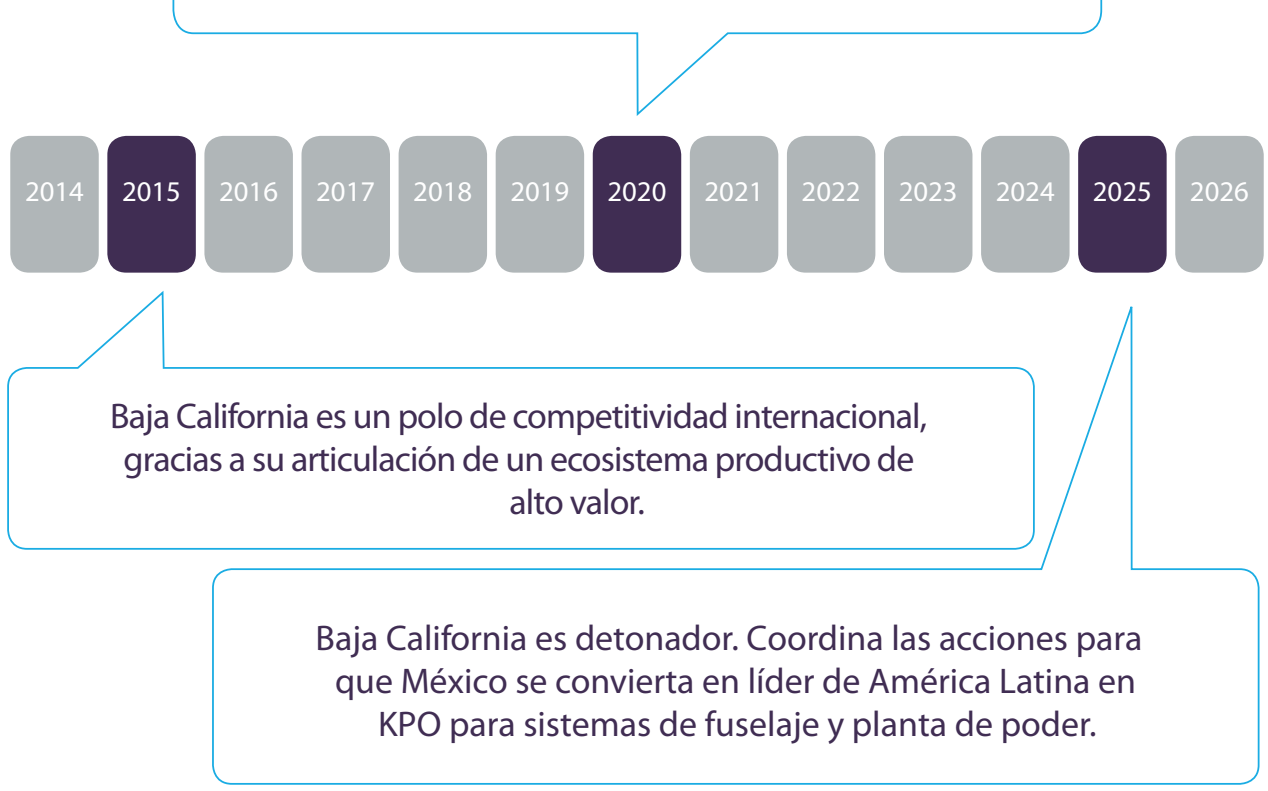

Figura 8. Hitos Estratégicos de una región mexicana (Baja California).

Fuente: Obregón (2014).

Los diferentes clústeres se encuentran integrados y estructurados a través de la Federación Mexicana de la Industria Aeroespacial, como se ilustra en la Figura 9., encabezada por un presidente y un personal de vicepresidentes que lidera cada una de las regiones en la nación (Figura 10.) (FEMIA, 2012).

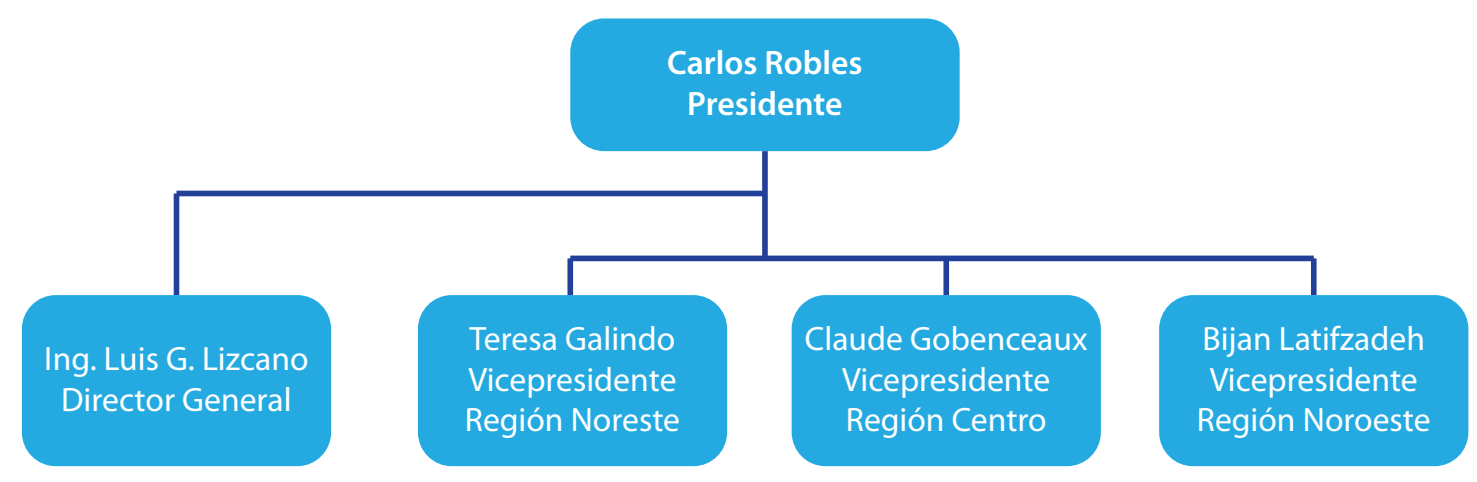

Figura 9. Estructura FEMIA.

Fuente: FEMIA (2012). 


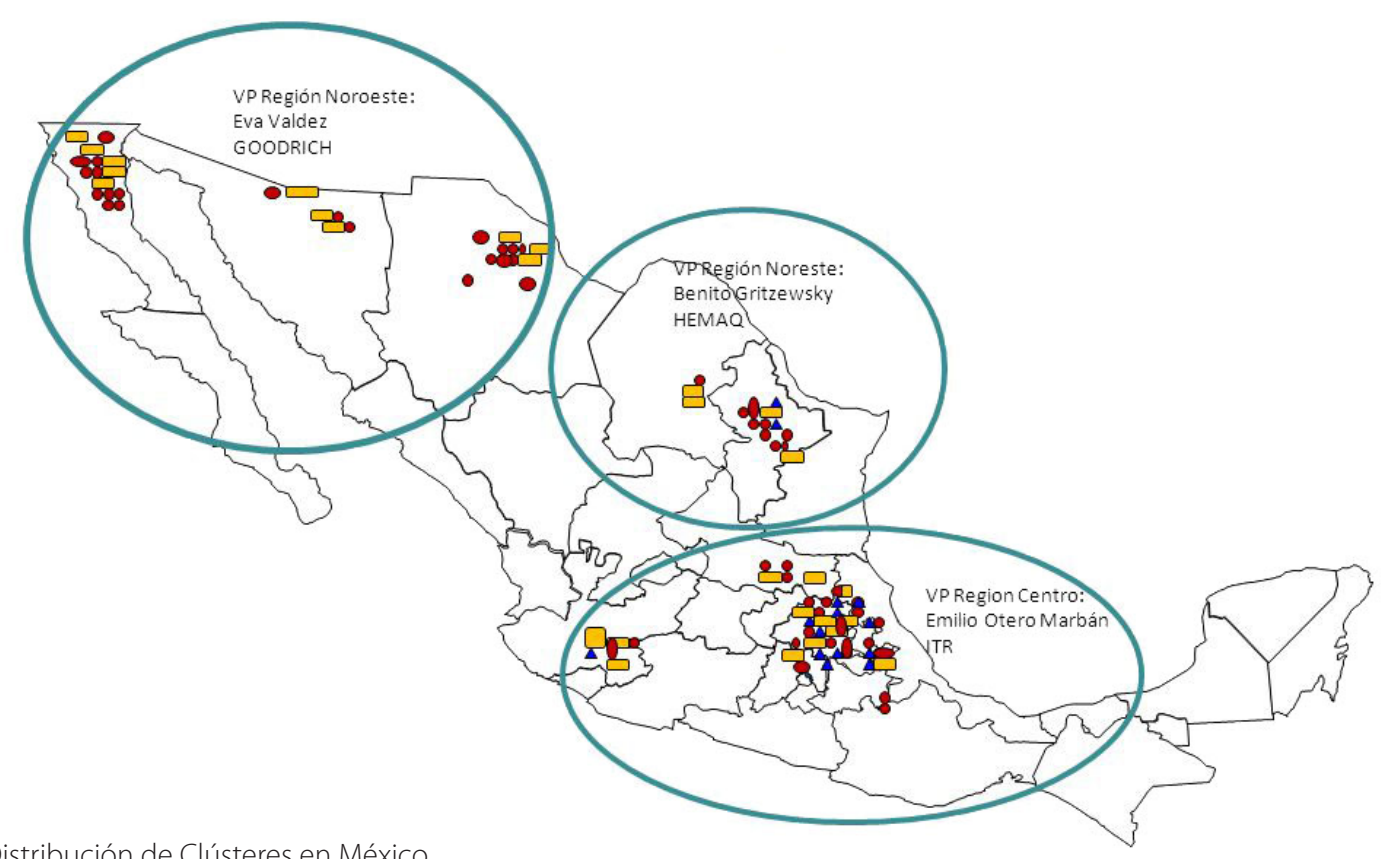

Figura 10. Distribución de Clústeres en México

Fuente: FEMIA (2012).

\section{Otros clústeres aeronáuticos internacionales:}

Clúster AEROCENTRE, Francia

Es importante identificar las fortalezas que presentan las diferentes regiones con el fin de enfocar los esfuerzos; muestra de ello es el caso del clúster francés de AEROCENTRE, el cual se especializó en los interiores de cabinas. Actualmente, el clúster está compuesto por 45 empresas, tiene con un total de 4.500 empleados y cuenta con 12 laboratorios de investigación (Normans Medioa, 2012). De igual forma, el clúster hace parte de GIFAS (Groupement des Industries Francaises Aeronautiques et Spatiales), área del Gobierno francés que promueve el desarrollo y crecimiento empresarial de la industria aeronáutica.

\section{Clúster de UAV, Reino Unido}

Dentro del desarrollo de un clúster, las alianzas son un factor determinante, ya que contar con socios estratégicos promueve la comercialización y la prestación de los servicios. Muestra de ello es el caso de este clúster, el cual ha presentado un gran desarrollo a raíz de la alianza generada con NAC (National Aeronautical Centre), dándose a conocer con clientes potenciales tanto en la parte militar, como en la parte civil. Así mismo, se benefició al crear alianzas con HUBs de mantenimiento y overhaul de aeronaves, permitiendo compartir servicios tecnológicos, know how, clientes, entre otros (Presswire, 2012).

\section{Clúster aeronáutico, Reino Unido}

Un estudio demostró que cuando una industria se encuentra localizada en un sector en donde operan empresas que laboran en un área afín, se tiende a tener crecimiento productivo mayor y a tener un promedio de generación de patentes alto (Bönte, 2004). Otro factor a considerar es la fuerza de cohesión que presente el clúster, el cual se mide mediante la eficiencia del flujo de información, la competitividad empresarial y el nivel de demanda (Bönte, 2004).

\section{Desarrollo aeronáutico nacional Clúster Aeroespacial Colombiano}

En la presente sección se realiza un diagnóstico del Clúster Aeroespacial Colombiano (C.A.C.), a través de una entrevista realizada con el señor Director Leonardo Mesa durante el desarrollo de la Feria de Capacidades realizada en el Comando Aéreo de Mantenimiento. Dicha entrevista tiene como finalidad determinar cuál es el estado actual del clúster (Mesa, 2016).

EI C.A.C. es una agrupación empresarial joven, conformada por 40 empresas, constituida desde el año 2011 y con la cualidad de que a nivel nacional es el primer clúster aeronáutico que se encuentra certificado por la Aeronáutica (REDCLUSTER, 
2012). Al ser un clúster certificado, entre otras ventajas, le ha permitido tener una mayor apertura comercial con el mercado nacional.

El clúster nació en Antioquia a partir del trabajo de un grupo multidisciplinario que tuvo como participante activo al Comando Aéreo de Combate No. 5, un sector empresarial de la industria antioqueña y universidades. Mencionada agrupación comenzó a llamar la atención de empresas, entidades gubernamentales e instituciones, al punto que actualmente está integrado por empresas de diferentes regiones (Antioquia, Costa Atlántica, Valle del Cauca y Risaralda).

La participación política regional, por intermedio de las Cámara de Comercio y otras instituciones de cada uno de los departamentos que conforman el clúster, ha generado un impacto positivo al representar un puente fundamental para el crecimiento y desarrollo industrial, así como también un medio de conexión entre el clúster y las industrias.

Al estar constituidos como un clúster, se han fortalecido las capacidades industriales, y con ellas, la adquisición de oportunidades de negocio, evidenciada en la obtención de avales para la ejecución de proyectos en convocatorias realizadas por Colciencias, Impulsa, Ministerio de defensa, entre otros.

La estrategia de trabajo desarrollada por el clúster se basa en la clasificación industrial según las capacidades de sus integrantes, de tal forma que se puedan orientar los diferentes proyectos aeronáuticos solicitados por los clientes; asimismo, realiza una sub-especialización con el fin de no redundar en los portafolios industriales ofrecidos.

El C.A.C. se ha puesto como meta realizar gestiones a nivel gubernamental para gestionar el crecimiento empresarial y la vinculación de otras industrias. Apoyados en el Ministerio de Industria y Turismo se alcanzó la eliminación del IVA para los elementos aeronáuticos importados, permitiendo una mayor competitividad al ofrecer servicios a costos más favorables.

El Ministerio de Defensa ha desarrollo el sector aeronáutico en el país a través de la implementación de los programas PINES (Proyectos Estratégicos de Nivel Nacional), enfocados en gestionar mejoras en el desarrollo, a través de la participación en proyectos del sector Defensa. Dicho plan se está implementando en la Armada Nacional y tiene como finalidad reducir de un $60 \%$ a solo un $10 \%$ el rubro asignado a compañías extranjeras para la fabricación de buques.
En cuanto a lo académico, el clúster ha integrado a las universidades mediante los centros de investigación. Desde su nacimiento, el clúster ha creado programas de vinculación con entidades educativas de alta calidad (Universidad de Antioquia, ITM, EAFIT, Escuela de Ingenieros). Una de las tareas asignadas a los centros de investigación son los procesos de ingeniería inversa para proyectos de sustitución de importaciones.

En lo referente al sector industrial, Avianca se encuentra realizando un mega proyecto que consiste en desarrollar un MRO (Maintance Repair and Overhaul). Dentro del presente proyecto, la empresa designó al C.A.C la producción de plataformas para el desarrollo de trabajos de mantenimiento.

La gestión de alianzas estratégicas a nivel internacional ha sido un objetivo por desarrollar; prueba de ello es la reciente alianza generada con el Clúster Mexicano, única agrupación externa a los Estados Unidos, que tiene certificación de la FAA. El convenio realizado con la agrupación industrial mexicana está enfocado en realizar procesos de acompañamiento, transferencia tecnológica, desarrollo de negocios, entre otros.

Enfocando el tema de relaciones globales a futuro, el C.A.C. se encuentra actualmente haciendo procesos de acercamiento con el Clúster Aeronáutico Español, con el fin de adquirir capacidades de certificación de servicios en el continente europeo, para la adopción de modelos tecnológicos. Otro cliente potencial, al cual se planea contactar, es el Clúster de Brasil, que en la última década ha desarrollado pasos agigantados por intermedio de su empresa Embraer.

\section{Participación del sector aeronáutico en Colombia}

El sector aeroespacial es un pilar fundamental para la nación ya que, considerando su compleja topografía, es en algunos casos el único medio de transporte disponible para conectar las regiones más apartadas, por lo que es el segundo medio más empleado en el 2015, con un registro del $11 \%$ (Figura 11.) (Ministerio de Transporte, 2015).

Cada día más personas en el país hacen uso del medio aéreo, alcanzando un crecimiento constante de $8 \%$ en la cantidad de pasajeros, desde el 2002 hasta el 2010. En el ámbito económico se ha evidenciado un alto crecimiento desarrollado entre el 2003 y el 2009, presentando un crecimiento financiero de $4.1 \%$ en el PIB (ATAC, 2008). 


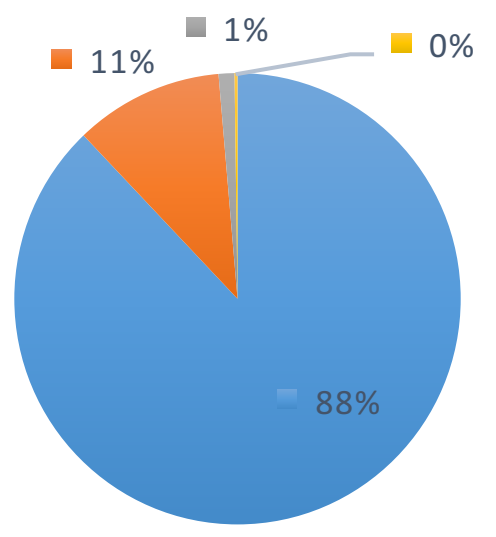

\section{- Terrestre Aéreo Eluvial Eerroviario}

Figura 11. Distribución por Medio de Transporte, Recuperado de Transporte en Cifras Estadísticas.

Fuente: Ministerio de Transporte (2016).

El gobierno colombiano ha generado iniciativas para mejorar el sistema de transporte aéreo mediante la inversión en modernización de la infraestructura; el acceso al mercado a aerolíneas internacionales (Aerorepública, LAN, ACES); la celebración de acuerdos multilaterales (Acuerdos de Cielos Abiertos); y la implementación de concesiones aeroportuarias. Una de las principales iniciativas fue la modificación estructural de la aviación civil, pasando de ser un departamento administrativo a una unidad administrativa especial, dependiente del Ministerio de Transporte.

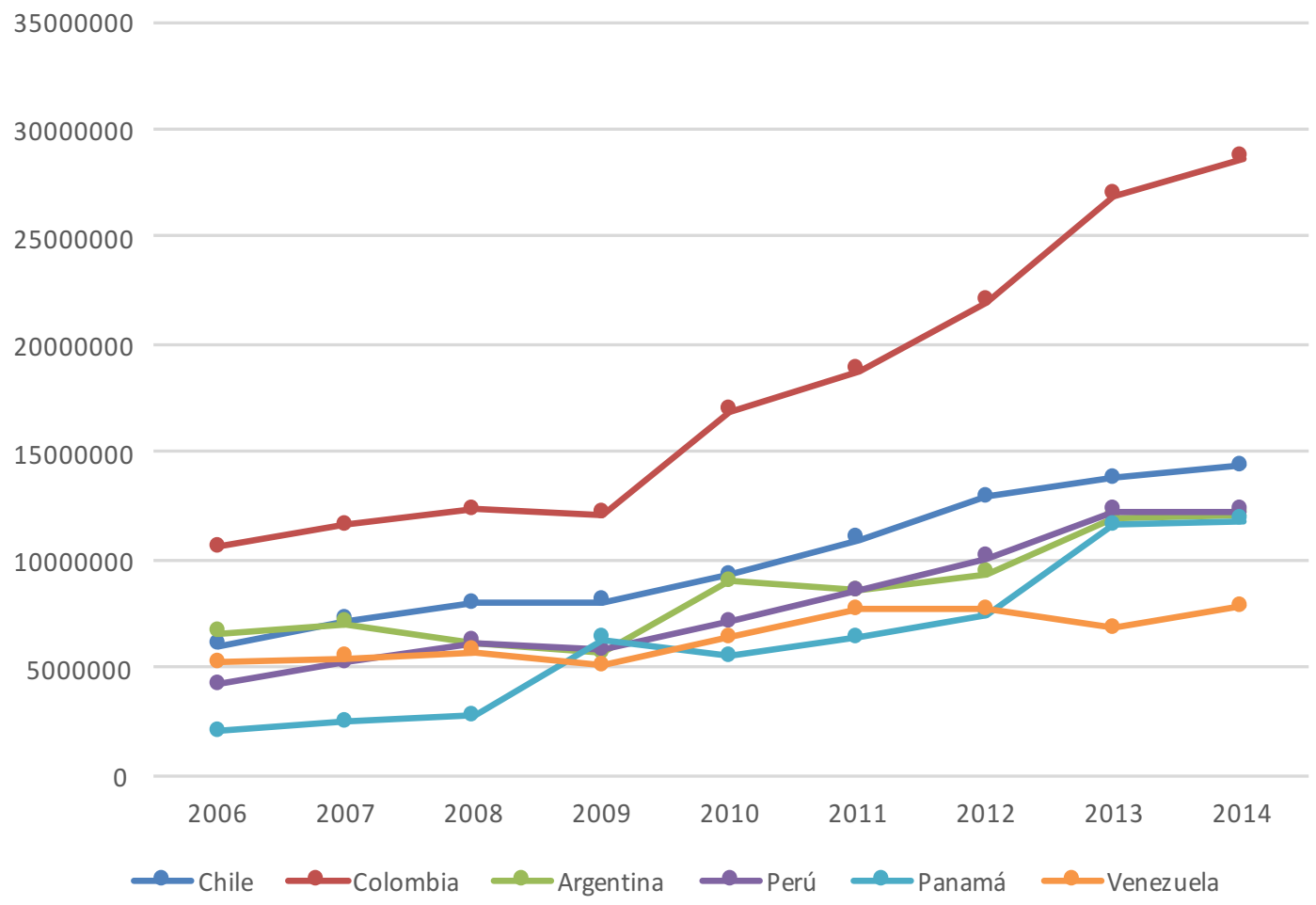

Figura 12. Cantidad de Pasajeros Transportados 2006-2014

Fuente: Banco Mundial (2014). 
Como se ilustra en la presente gráfica, la aviación nacional ha presentado un alto posicionamiento dentro del sector latinoamericano, siendo este un referente en la región (Banco Mundial, 2014). Otro factor del transporte que es importante analizar es el aumento en la cantidad de pasajeros que se ha presentado a nivel nacional en los últimos 8 años, evidenciando un crecimiento del 100\% (Mauricio Olivera, 2011).

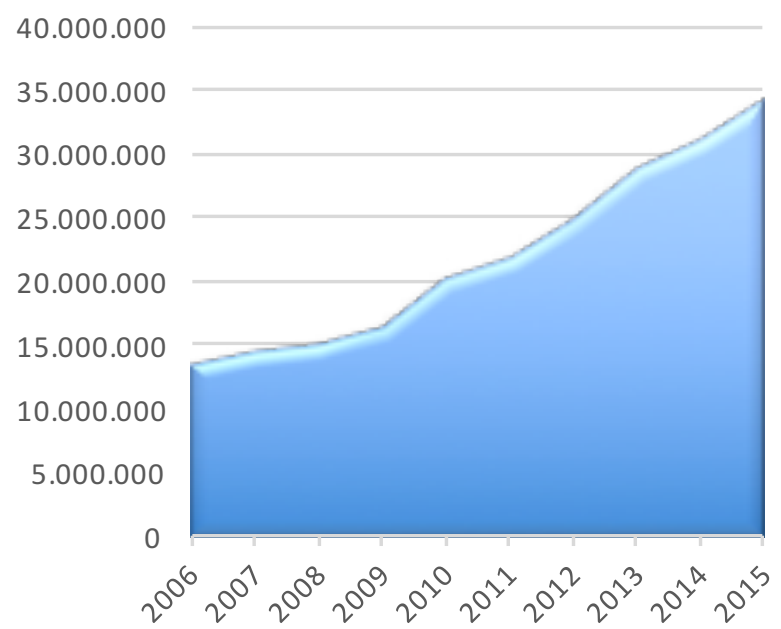

Figura 13. Serie Anual de Pasajeros y Carga Fuente: Aerocivil (2016).

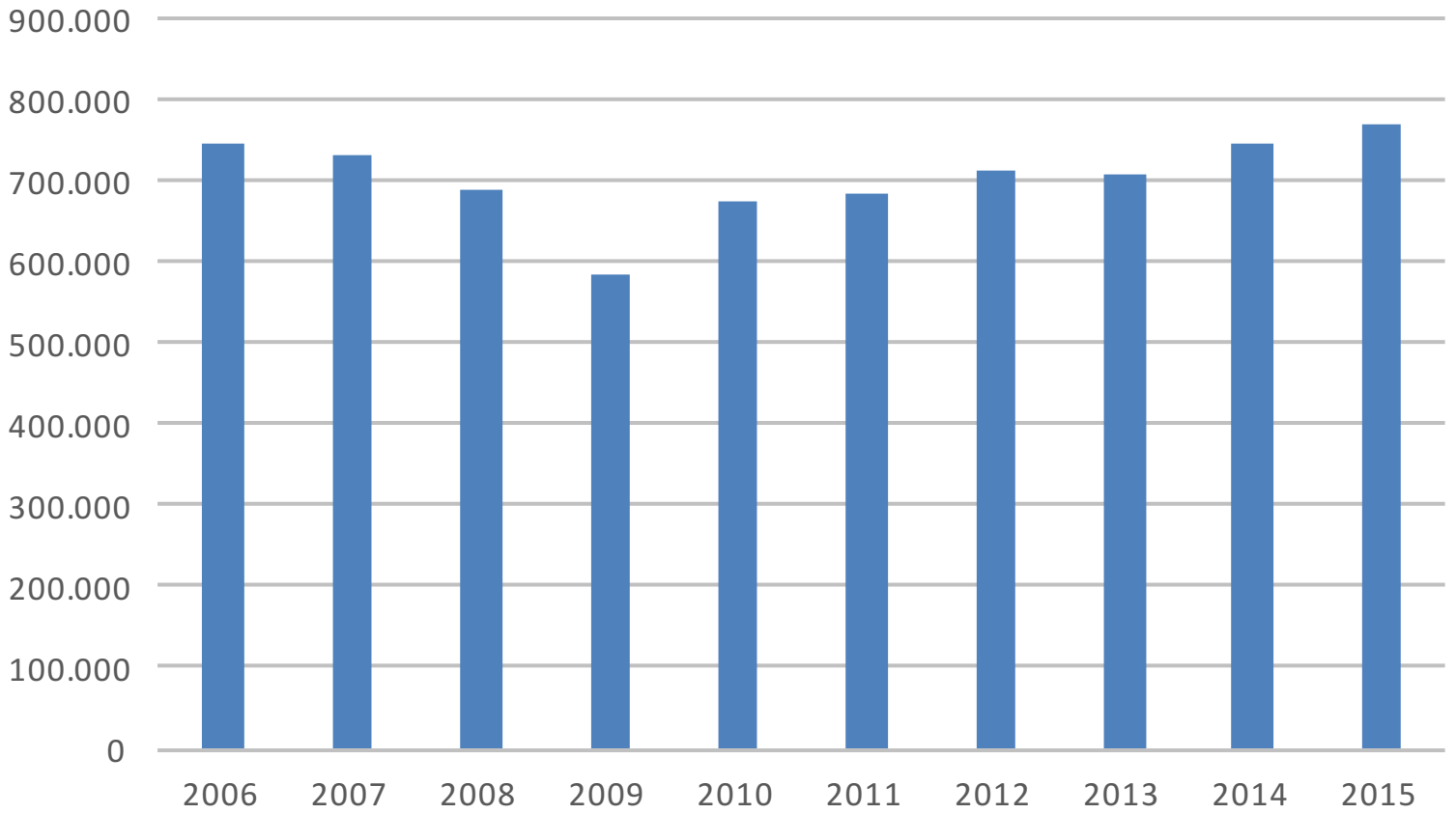

Figura 14. Serie Anual de Pasajeros y Carga

Fuente: Aerocivil (2016). 
CIENCIA Y PODER AÉREO | ISSN 1909-7050 | E-ISSN 2389-9468 | Vol. 12 | Ene - Dic 2017 | Escuela de Postgrados de la Fuerza Aérea Colombiana | pp 36-58

Analizando el índice de transporte de pasajeros y carga desde un ámbito nacional, se evidencia (Figura 13 y 14) el crecimiento en cuanto a la cantidad de pasajeros y toneladas transportadas, tanto a nivel nacional, como internacional (Aerocivil, 2016). Dichos comportamientos son un claro reflejo del aumento considerable en la demanda que se ha venido presentando.

Otro factor importante es el alto crecimiento que ha tenido el transporte internacional, lo cual se traduce en una mejora en la economía nacional a través del turismo y la expansión hacia mercados mundiales (ICAO, 2009). En el ámbito del transporte de carga, se evidencia una tendencia oscilatoria que refleja la necesidad de implementar estrategias para generar productos de calidad, cambiando la tradicional exportación de materia prima por productos tratados.

Es importante resaltar de igual forma que la aviación es un mercado que genera efectos inducidos, directos e indirectos, trayendo consigo, entre otros aspectos, beneficios económicos, adquiridos mediante la generación de contrataciones (OMC, 2005). En el siguiente gráfico se ilustran los porcentajes de inversión financiera del sector aeroespacial.

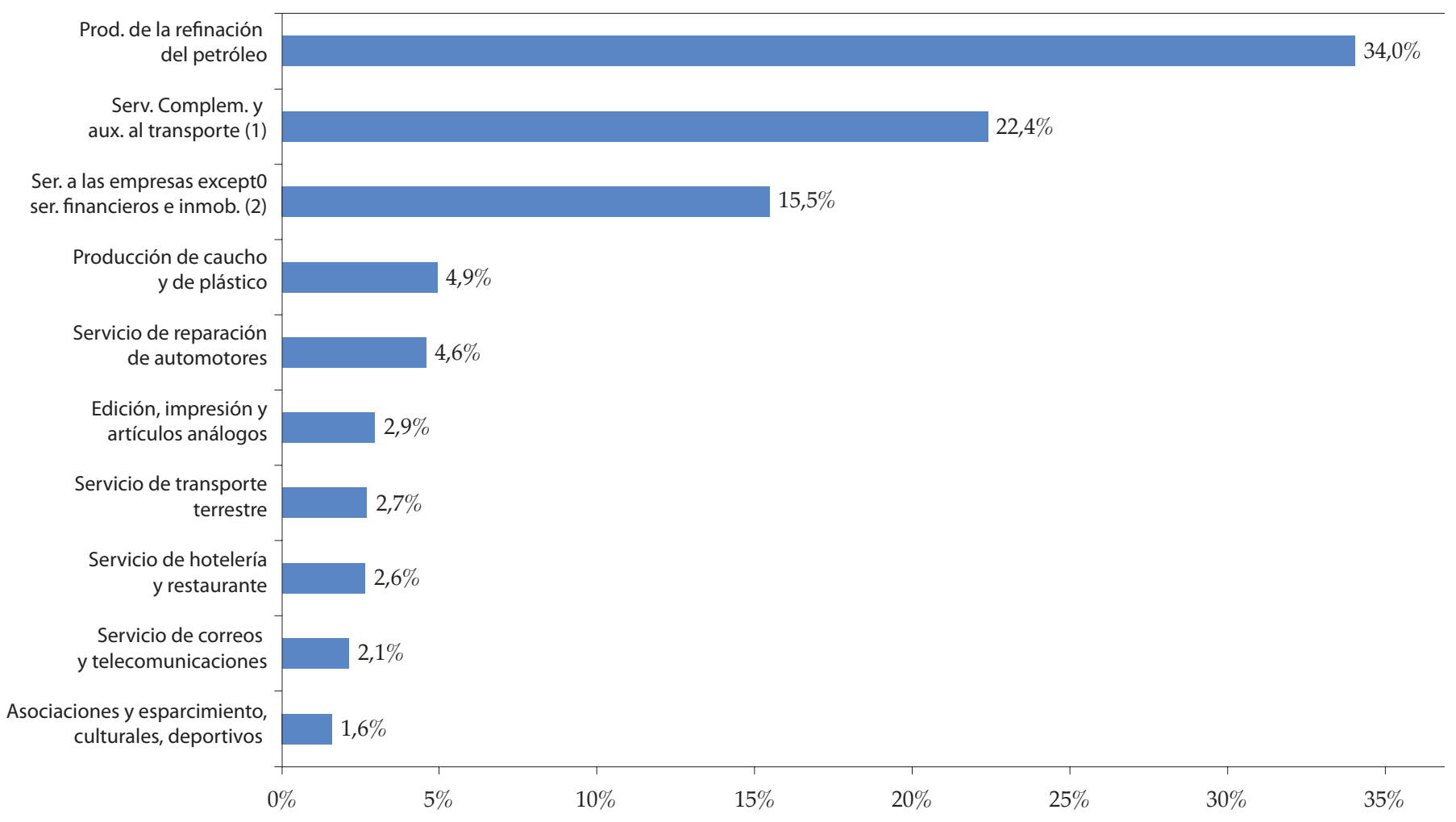

Figura 15. Compras intermedias del sector de servicios de transporte aéreo.

Fuente: Olivera (2011).

El DANE reportó en el año 2007 un consumo de 4.2 billones de pesos del sector aeronáutico en Colombia, referente al efecto directo, generó 931 mil millones de pesos al sector trabajo, 1,5 billones al sector capital y paga impuestos por 77 mil millones de pesos (Mauricio Olivera, 2011). 


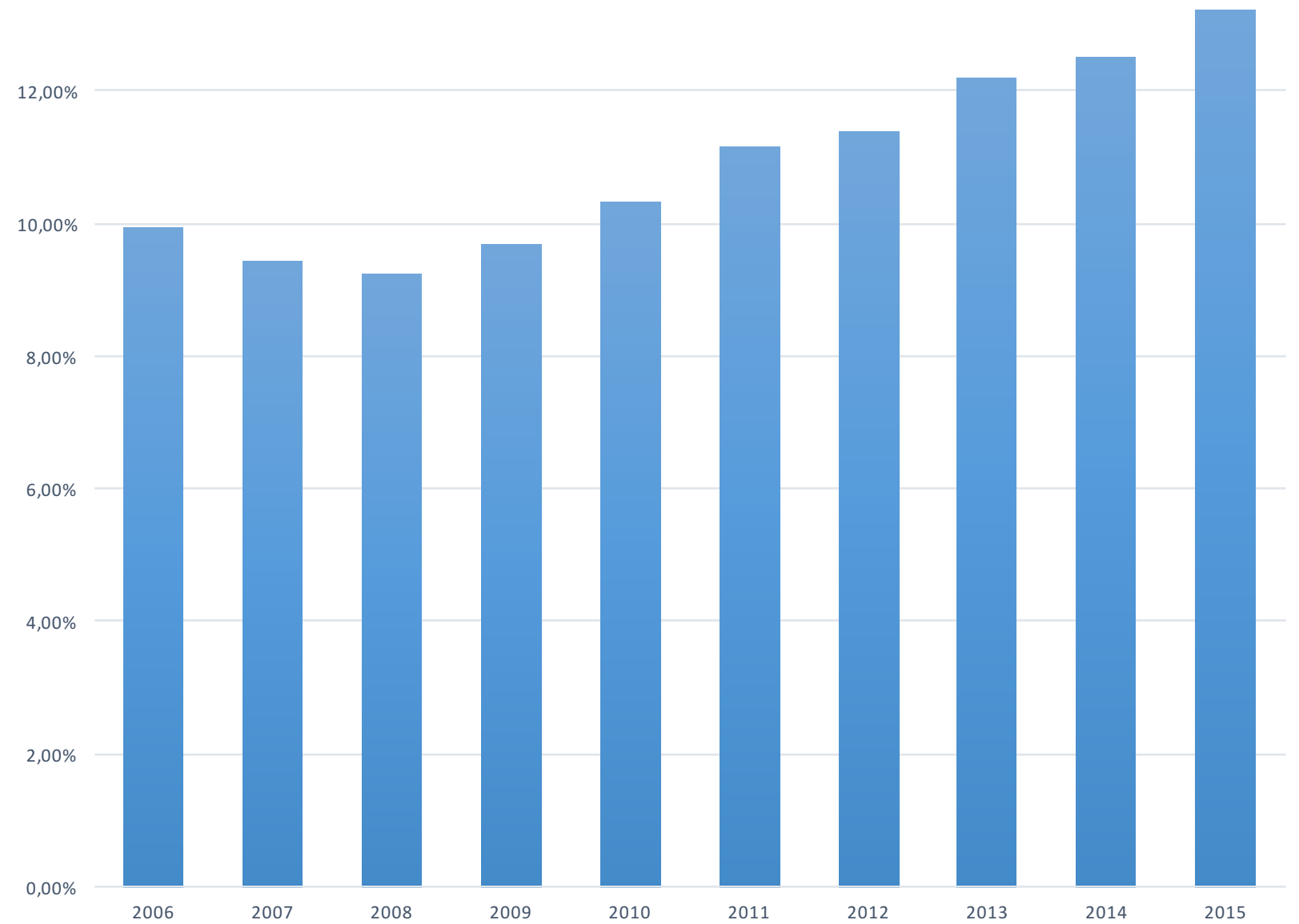

Figura 16. Participación del PIB de transporte aéreo en el PIB Nacional.

Fuente: Ministerio de Transporte (2016).

En la figura 17 se identifica la participación que ha tenido el sector de la aviación en el PIB, presentando un incremento de 3.27\% en los últimos años (2006 - 2016). Se logra evidenciar la constante evolución que ha venido presentando el sector en lo referente a la productividad financiera (Ministerio de Transporte, 2015). 
Factores claves para el éxito del C.A.C.

Tabla 4.

Síntesis objetivo específico No. 1

\begin{tabular}{|c|c|c|c|}
\hline Característica & Clúster Español & Clúster Brasil & Clúster Mexicano \\
\hline Estructura & \multicolumn{3}{|c|}{$\begin{array}{l}\text { Como se logró evidenciar en el presente artículo, hoy en día los clústeres aeroespaciales cuentan } \\
\text { con una estructura como la relacionada en la figura } 1 .\end{array}$} \\
\hline Organización & $\begin{array}{l}\text { Está basada en patronatos } \\
\text { multidisciplinarios, confor- } \\
\text { mados por los principa- } \\
\text { les promotores del sector } \\
\text { aeroespacial (industria, } \\
\text { academia, Gobierno, aso- } \\
\text { ciaciones empresariales, } \\
\text { cámaras de comercio, sin- } \\
\text { dicatos, entre otros). }\end{array}$ & $\begin{array}{l}\text { La organización de las empre- } \\
\text { sas se encuentra administrada } \\
\text { por el Gobierno a través de la } \\
\text { Administración Nacional de } \\
\text { Aviación Civil, la cual gestiona } \\
\text { la asignación de certificados } \\
\text { aeronáuticos. }\end{array}$ & $\begin{array}{l}\text { Los clústeres se encuentran lidera- } \\
\text { dos por la Federación de la Indus- } \\
\text { tria Aeroespacial, encabezada por } \\
\text { un presidente y vicepresidente } \\
\text { por cada una de las regiones ae- } \\
\text { roespaciales. }\end{array}$ \\
\hline
\end{tabular}

Fuente: Elaboración propia.

Tabla 5.

Síntesis objetivo específico No. 2

\begin{tabular}{|c|c|c|c|}
\hline Eje & Clúster Español & Clúster Brasil & Clúster Mexicano \\
\hline Gubernamental & $\begin{array}{l}\text { - Implementación de ini- } \\
\text { ciativas de desarrollo } \\
\text { competitivo. } \\
\text { - } \quad \text { Disposición de infraes- } \\
\text { tructura aeroportuaria } \\
\text { - Generación de progra- } \\
\text { mas nacionales } \\
\text { Organización basada en } \\
\text { patronatos }\end{array}$ & 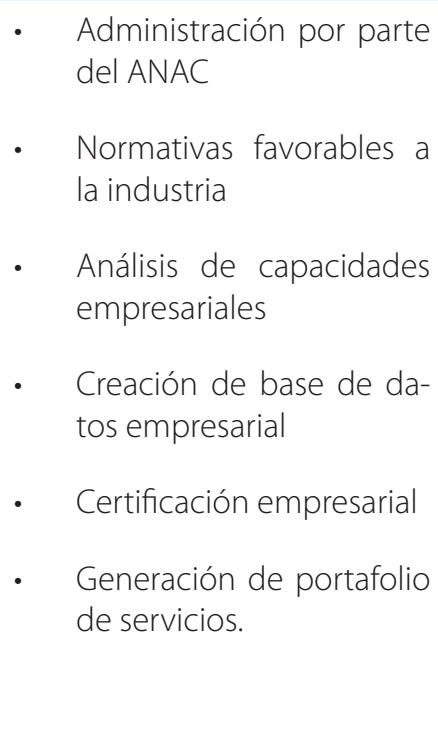 & $\begin{array}{l}\text { - } \quad \text { Liderado por la Agencia Espa- } \\
\text { cial Mexicana } \\
\text { - } \quad \text { Acuerdos de cooperación es- } \\
\text { tratégicos } \\
\text { - } \quad \text { Tratado de comercio cómodo } \\
\text { y seguro } \\
\text { - } \quad \text { Metas estratégicas (PVN) } \\
\text { - Certificación, } \\
\text { - } \text { SNC. } \\
\text { Ingreso arreglo Wassenaar } \\
\text { - Creación ZEE } \\
\text { - Simplificación Arancelaria }\end{array}$ \\
\hline Industrial & $\begin{array}{l}\text { - } \quad \text { Generación de alianzas } \\
\text { - } \quad \text { Cooperanización piramidal } \\
\text { - Gestión del flujo de infor- } \\
\text { mación }\end{array}$ & $\begin{array}{l}\text { - Adquisición de capacida- } \\
\text { des mixtas } \\
\text { - } \quad \begin{array}{l}\text { Organización piramidal } \\
\text { según capacidades }\end{array}\end{array}$ & $\begin{array}{l}\text { - Sofisticación tecnológica } \\
\text { - } \quad \text { ompetitividad en la mano de } \\
\text { obra } \\
\text { - Vinculación AFRA }\end{array}$ \\
\hline Académico & $\begin{array}{l}\text { Creación de foros multi- } \\
\text { disciplinarios }\end{array}$ & Centros de investigación & $\begin{array}{l}\text { Normatividad en certificación } \\
\text { Integración instituciones }\end{array}$ \\
\hline
\end{tabular}

Fuente: elaboración propia. 


\section{Conclusiones}

\section{Mejores prácticas gubernamentales}

Es importante disponer de una infraestructura aeroportuaria localizada de manera estratégica con el fin de promover la comercialización y la ejecución de servicios aeroespaciales.

Asimismo, es necesario que se cree un liderazgo del Gobierno para determinar las capacidades y campos de acción de cada una de las empresas certificadas, de tal forma que se garantice que las empresas presten servicios para los cuales se encuentran capacitados, además de generar una base de datos que permita facilitar la comercialización con clientes potenciales que requieran trabajos de mantenimiento tanto a nivel nacional como internacional.

En esta línea, una mejor práctica sería gestionar de parte del Gobierno colombiano la creación de una entidad gubernamental que se encargue exclusivamente de desarrollar el sector aeroespacial aviación, considerando que la Aerocivil actualmente desarrolla múltiples roles que, en muchos casos, desvían su atención del objetivo de fomentar el crecimiento industrial en la nación.

La entidad gubernamental a cargo del desarrollo aeroespacial debe tener también la indispensable tarea de impulsar importantes acuerdos estratégicos con entidades internacionales, para promover tratados de exportación cómodos y seguros.

Considerando que Colombia está ubicando en el continente americano, el Gobierno debe abundar esfuerzos para generar un acuerdo bilateral que logre generar un reconocimiento de parte de la FAA para la certificación de partes, componentes, sistemas aeronáuticos, desarrollo de procesos de mantenimiento y reparación (MRO), entre otros.

El Clúster Aeroespacial Colombiano viene realizando trabajos principalmente con la Fuerza Aérea Colombiana, ya que el sector Defensa es bastante tentativo y ofrece proyectos interesantes de innovación y desarrollo. Sin embargo, si se desea abarcar mercados militares internacionales es necesario que el Gobierno contribuya a la seguridad y estabilidad mediante la generación de sistemas de control de importaciones, que dan credibilidad y confianza a otras naciones para comercializar material bélico en el mercado colombiano.

Colombia ya viene generando negociaciones Offset con diferentes proveedores a nivel mundial; sin embargo, se debe continuar fomentando dicho tipo de convenios ya que mejora la comercialización al atraer nuevas tecnologías, promover el desarrollo industrial y comercial, y explotar la competitividad de los proyectos estratégicos nacionales.
De la mano con este ideal de los centros integrales, se recomienda innovar mediante la creación de parques especializados, que tengan como finalidad facilitar la transacciones y logística de las empresas que funcionen en su interior, y que desarrollen procedimientos estándar alineados con los regímenes internacionales de control.

Dentro de las estrategias claves que debe implementar el Gobierno con el fin de favorecer el progreso del sector aeroespacial en materia de importación, se recomienda gestionar la importación temporal de bienes destinados a la elaboración, transformación o reparación de mercancía de procedencia extranjera para su exportación, sin cubrir el pago de impuestos por el ingreso al país; y que se realice una clasificación que, según el tipo de material importado, se tenga la opción por parte de la empresa de recuperar el importe del impuesto pagado.

Pasando al tema del sector arancelario, y teniendo como premisa llamar la atención de mercados internacionales, el Gobierno debe implementar medidas que permitan realizar una simplificación arancelaria, un planteamiento de esquemas de excepción y la creación de fracciones arancelarias, de tal forma que se facilite la comercialización de productos y materia prima para los procesos de producción y manufactura.

Otra estrategia que puede atraer mercados globales está relacionada con la generación de zonas económicas especiales, que ofrezcan incentivos a los inversionistas externos mediante altas expectativas económicas, exenciones fiscales, condiciones favorables de infraestructura, facilidades administrativas, mano de obra calificada, entre otros.

La autoridad aeronáutica colombiana debe generar una base de datos de todas las empresas colombianas que ofrecen servicios aeronáuticos, tanto en materia de producción como de mantenimiento. Asimismo, es importante que estipule un proceso de certificación que permita clasificar los servicios especializados que presta cada una de las entidades oferentes, de tal forma que se tenga un portafolio definido de las capacidades nacionales.

\section{Mejores prácticas industriales}

Como es sabido a nivel mundial, el tema de la calidad es hoy un requisito indispensable para cualquier industria, y el sector aeroespacial colombiano no puede ser ajeno a esta tendencia, es por esto que se recomienda contar con un ente gubernamental que garantice altos estándares de calidad para grupos industriales que conforman el sector aeroespacial colombiano. Dicha entidad tiene la importante misión de realizar las gestiones necesarias para adminis- 
CIENCIA Y PODER AÉREO | ISSN 1909-7050 | E-ISSN 2389-9468 | Vol. 12 | Ene - Dic 2017 | Escuela de Postgrados de la Fuerza Aérea Colombiana | pp $36-58$

trar la certificación, acreditación e implementación de mejores prácticas, que constituyen la base para la generación de empresas de alta calidad, para satisfacer el complejo mercado internacional.

La triple alianza, mencionada anteriormente (Gobiernoacademia-industria), debe trabajar de manera mancomunada en la creación de centros integrales que brinden servicios aeronáuticos mediante un modelo de ecosistema que desarrolle la industria mediante ventajas en el mantenimiento, establecimiento de zonas comerciales preferenciales y formación de las diferentes áreas del sector aeroespacial.

Alineado con las políticas internacionales sobre cuidado del medio ambiente, el clúster aeroespacial debe general directrices que estimulen la implementación de modelos de producción, que permitan reutilizar las piezas de las aeronaves una vez cumplan su ciclo de vida útil. De igual forma, la presente iniciativa tiene implicaciones en la reducción de costos de manufactura y consecución de material, para temas de producción.

El flujo de información existente en el interior del Clúster Aeroespacial Colombiano es un factor crítico, ya que de ello depende la agilidad y precisión con las cuales se realiza la transferencia de datos entre las empresas participantes. La anterior observación se realiza considerando el crecimiento evidente que ha presentado el clúster, ya que inició sus primeros pasos en Antioquia y actualmente se encuentra constituido por empresas del Valle del Cauca y la Costa Atlántica. El flujo de información también debe ser un tema de analizar al momento de realizar convenios con agrupaciones empresariales aeronáuticas internacionales.

\section{Mejores prácticas académicas}

A través del apoyo de las instituciones educativas, se recomienda generar espacios académicos mediante la generación de foros que impulsen la investigación, la innovación y el desarrollo académico del clúster. Lo anterior considerando que de esta forma se logra atraer profesionales de múltiples disciplinas relacionadas con el sector aeroespacial, para generar espacios que permitan compartir con conocimientos, experiencias y discusiones acerca del modo de implementar una industria aeronáutica en la nación

Es necesario que Colombia se convierta en un destino atractivo para la innovación y eficiencia operativa, lo cual se logra mediante el aporte de la academia para crear una alta riqueza y disponibilidad de talento humano profesional en áreas de ingeniería y afines. Lo anterior debe apuntar a ofrecer mano de obra para procesos de manufactura aeroespacial de alta calidad y bajo costo.

\section{Referencias}

Aerocivil. (2016). Serie anual Pasajeros y Carga. Recuperado de http://www.aerocivil.gov.co/atencion/estadisticas-de-lasactividades-aeronauticas/aeropuertos/forms/allitems.aspx

Agencia Espacial Mexicana (AEM) (2010). Catalogo y Análisis de Capacidades de Investigación y Desarrollo Tecnológico Espacial en México. Recuperado de http://www.gob.mx/aem

Agencia Nacional de Aviación Civil ANAC. (2005). Registro Aeronáutico Brasileñol. Recuperado de: http://www.anac.gov.br/ assuntos/sector-regulado/aeronaves/rab/copy_of_rab

Aguilera, C. M. (2010). Past, present and future of the andalusian aeronautical cluster, España: Network-Centric Collaboration.

Airbus. (2009). Airbus demonstrates 40 years of innovation and shaping efficiency. Recuperado de http://search.proquest. com/docview/446139790?accountid=143348

Aircraft Fleet Recycling Association AFRA. (2005). The AFRA BMP. Recuperado de http://afraassociation.org/accreditation/theafra-bmp/

Al Bawaba, A.B. (2015). MENA Report. Recuperado de http://search. proquest.com/docview/1662312126? accountid $=143348$

Anand, G, \& Kodali, R. (2008). Benchmarking the benchmarking models. Emerald Group Publishing Limited, 15(3), 257 - 291.

Antonelli, A.C. (1999). Collective Knowledge Communication and Innovation: The evidence of technological districts. Regional Studies, 34(6), 535-547.

Ariño, A.A \& Mikhail, M. A, Irina, I.S, Irina, I.R \& Joaquim, J.V. (1997). Partner selection and trust building in west European-Russian joint ventures: a western perspective. International Studies of Management \& Organization, 27(1), 19-37.

ATAC, A. D. (2008). Estudio económico del transporte aéreo en Colombia 1970 - 2006. Asociación del transporte aéreo en colombia-ATAC. Bogota, Colombia.

Bönte, W. (2004). Innovation and Employment Growth in Industrial Clusters: Evidence from Aeronautical Firms in Germany. International Journal of the Economics of Business, 11(3), 259-278.

Brazilian Civil Aviation (2003). Brazilian Civil Aviation Regulation RBHA 135. Brasilia: Brazilian Regulation.

Carrasco, O. V. (2009). SCIELO. Recuperado de http:// www.scielo.org.bo/scielo.php?script=sci_arttext\&pid $=$ S1726-89582009000100010

Carrillo, A. H. (2005). Diagnóstico de la industria aeroespacial en Baja California. Características productivas y requerimientos actuales y potenciales de capital humano. Baja California, México: El Colegio de la Frontera Norte. 
Comercio y Aduanas. (2001). Programa de comercio exterior drawback. Recuperado de http://www.comercioyaduanas. com.mx/comercioexterior/programas/66-programas/142programa-drawback

CONACIT. (1970). Consejo Nacional de Ciencia y Tecnología. Recuperado de http://conacyt.gob.mx/

Cooke, K. M. (1998). The associational economy: firms, regions, and innovation. Oxford, England: Oxford University Press.

De Bruecker, P, Van Den Bergh, J. (2015). A model enhancement heuristic for building robust aircraft maintenance personnel rosters with stochastic constraints. European Journal of Operational Research, 246(2) 661-673

Durand, J. M. (2008). Aircraft maintenance organizational structure changes (Master's thesis). Air Force institute of technology. Ohio, United States of America

Economía, S. d. (2006). Industria manufacturera, maquiladora y de servicio de exportación. Recuperado de http://www.20062012.economia.gob.mx/comunidad-negocios/industria-ycomercio/instrumentos-de-comercio-exterior/immex

El Grupo del Banco Mundial (2014). El Grupo del Banco Mundial. Recuperado de http://datos.bancomundial.org/indicador/ IS.AIR.PSGR/countries/CO-CL-AR-PE-PA-VE?display=graph

FAA, 2. (2009). Certification procedures for products and articles. Recuperado de http://www.ecfr.gov/cgi-bin/textidx?node=14:1 $0.1 \cdot 3.9$

FAA, 3. (2002). Airworthiness directives. Recuperado de http:// www.ecfr.gov/cgi-bin/text-idx?node=14:1.0.1.3.20

FEMIA. (2012). Federación Mexicana de la Industria Aeroespacial. Recuperado de http://www.femia.com.mx/index. php? module $=$ femia\&section $=2$

García, J. A. (2005). Clústers. Recuperado de http://www.monografias.com/trabajos29/clusters/clusters.shtml

Geuna, A. A, Aldo, A.G (2001). Does proximity matter for knowledge transfer from public institutes and universities to firms? (Research) University of Sussex. London, England.

Goldstein, A. (2002). Embraer: De campeón nacional a jugador global. Revista CEPAL, 77, 101-121

González, R. M. (2014). Es posible el éxito de las zonas económicas especiales. Forbes. Recuperado de https://www.forbes.com. $\mathrm{mx} /$ posible-exito-las-zonas-economicas-especiales/.

Government of Mexico, G. o. (2003). Agreement between the Government of the united states of America. Recuperado de http:// www.sct.gob.mx/fileadmin/DireccionesGrales/DGAC-archivo/modulo2/us-mexico-ata-final-english-102115.pdf.iloi

Group, T. A. (2013). Mexico se une al groupo de Australia. Recuperado de http://www.australiagroup.net/es/mexico_ga.html
HELICE. (2016). HELICE, Andalucian Aerospace Cluster. Recuperado de http://helicecluster.com/es/page/organizacion-yfuncionamiento.htm

ICAO. (2009). Overview of trends and developments in international air transport. ICAO Secretariat. Recuperado de https://www. icao.int/sustainability/Documents/OverviewTrends.pdf

ICAO, 8. (2010). Airworthiness of aircraft. ICAO Secretariat. Recuperado de http://code7700.com/pdfs/icao_annex_8.pdf

Ingram, L. A. (2000). Knowledge Transfer in Organizations: Learning from the Experience of Others. Organizational Behavior and Human Decision Processes. 82(1), 1-8.

Jorge, J.R, Claudia, C.S, Marisol, M.A. (2015). Transporte en cifras estadísticas. Bogotá: Ministerio de Transporte.

Kehayas, N. (2007). Aeronautical technology for future subsonic civil transport aircraft. Aircraft Engineering and Aerospace Technology. 79(6), $600-610$

Liu, W. K. (2009). Advantage competition of inter-partner learning in international strategic alliance. Journal of Global Business Issues, 3(2), 123.

Machado, C. (Julio de 2013). Clustering the technical capability in the brazilian aeronautical maintenance industry. Recuperado de http://search.proquest.com/docview/1505321583?ac countid $=143348$

Maldonado, A. G. (2009). Parques industriales de México. Comercio Exterior. Recuperado de http://revistas.bancomext.gob. mx/rce/magazines/122/6/RCE6.pdf

Media, N. (2012). AEROCENTRE. Recuperado de: http://search.proquest.com/docview/926944023?accountid $=143348$

Mesa, L. (Junio de 2016). Diagnóstico Clúster Aeroespacial Colombiano. (A. Bello, Entrevistador). Comunicación personal.

Ministerio de Transporte. (2010). Decreto 2937 del Ministerio de Transporte. Recuperado de https://www.secad.mil.co/sites/ default/files/Decreto\%202937_05\%2008\%202010.pdf

Mona V. Makhija, K. K. (1997). Measuring globalization of industries using a national industry approach: Empirical evidence across five countries and over time. Journal of International Business Studies.

Mora, L. A. (2013). Universidad Mariana. Recuperado de http:// www.umariana.edu.co/EditorialUnimar/art-revision.pdf

Nohora Eugenia, Y. C. (2002). OFFSETS: Aproximación teórica y experiencia internacional. Bogota: Departamento Nacional de Planeación.

NSG. (2013). Nuclear Suppliers Group. Recuperado de: http:// www.nuclearsuppliersgroup.org/es/

Obregón, Á. (2014). Industria aeroespacial mexicana mapa de ruta 2014. Ciudad de México: D.R. @ProMéxico. 
CIENCIA Y PODER AÉREO | ISSN 1909-7050 | E-ISSN 2389-9468 | Vol. 12 | Ene - Dic 2017 | Escuela de Postgrados de la Fuerza Aérea Colombiana | pp 36-58

OMC. (2005). Informe sobre el comercio mundial. Recuperado de https://www.wto.org/spanish/res_s/booksp_s/anrep_s/ world_trade_report05_s.pdf

Olivera, M, Cabrera, P, Bermudez, W, \& Hernandez, A (2011). El impacto del transporte aéreo en la economía colombiana y las políticas públicas. Cuadernos de FEDESARROLLO. Recuperado de http://hdl.handle.net/11445/165

Ornelas, S. (2007). Sonora the largest aerospace cluster integrated in Mexico. Mexico's Aeronautical industry takes off. Mexico Now, $42-46$.

Otero, J. (2015). Aplicación de un modelo de regresión a análisis de los conductos de transferencia tecnológica del cluster aeroespacial andaluz. Sevilla: Universidad de Sevilla.

PIMA. (2005). Programme for innovation and modernisation of andalusia. Sevilla: Consejería de Innovación Ciencia y Empresa.

Presswire, M. (2012). Normans Media Ltd. Recuperado de http:// search.proquest.com/docview/1232215089?account $\mathrm{id}=143348$

PROMEXICO. (2007). PROMEXICO Inversión y Comercio. Recuperado de http://www.promexico.gob.mx/es/mx/mapas-de-ruta

RAC, 2. (2015). Certificación de aeronaves y componentes de aeronaves. Bogotá: Aeronáutica Civil. Recuperado de: http:// www.aerocivil.gov.co/AAeronautica/Rrglamentacion/RAC/ Biblioteca\%20Indice\%20General/RAC\%20\%2021\%20-\%20 Certificaci\%C3\%B3n\%20de\%20aeronaves\%20y\%20componentes\%20de\%20aeronaves.pdf

REDCLUSTER. (2012). CEASCOL - Cluster Aeroespacial Colombiano. Recuperado de http://www.redclustercolombia.com/ clusters-en-colombia/iniciativa/130

Rosenfeld, S. (1997). Bringing business clusters into the mainstream of economic development, European Planning Studies. European Planning Studies, 5(1), 3-23.

Secretaría, O. d. (2012). México ingresa al Arreglo de Wassenaar para bienes de uso dual. Recuperado de http://www.economia-snci.gob.mx/sic_php/pages/bruselas/trade_links/esp/ eneesp2012.pdf

UAEAC. (2014). Plan de navegación aérea. Recuperado de http:// www.aerocivil.gov.co/Aerocivil/PlanGestControl/PoliticasPlanesProy/Programas\%20y\%20Proyectos\%20en\%20Ejecucion/ PNA\%20COL\%20VOL\%201\%20v07\%20\%20borrador.pdf

WestWorld. (28 de Mayo de 2014). SlideShare. Recuperado de http://www.slideshare.net/westworldconsulting/webinaraccessing-the-european-aerospace-market-and-supply-chain 


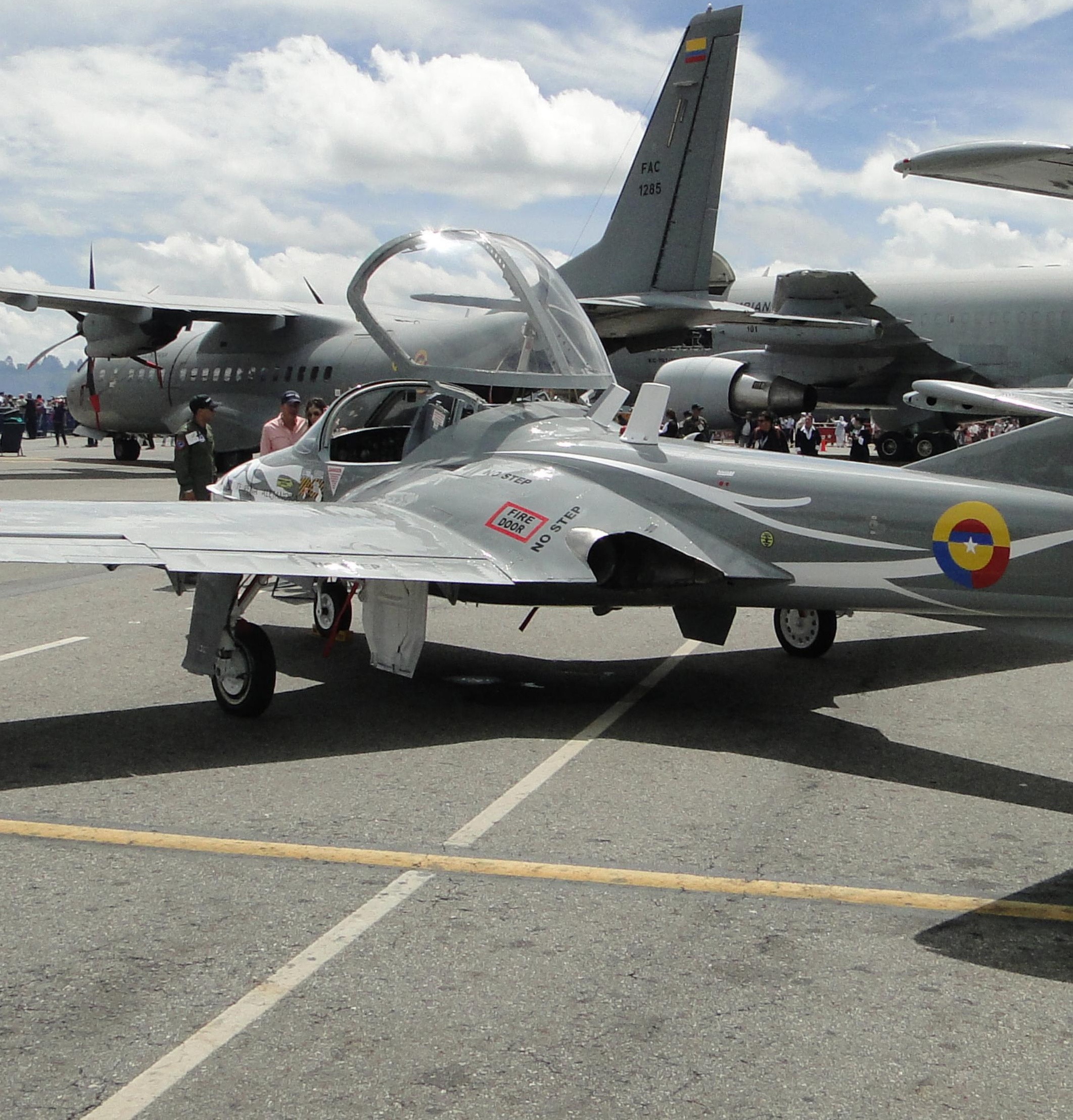

